\title{
Optimal Implementation of Simulink Models on Multicore Architectures with Partitioned Fixed Priority Scheduling
}

\author{
Shamit Bansal \\ Thesis submitted to the Faculty of the \\ Virginia Polytechnic Institute and State University \\ in partial fulfillment of the requirements for the degree of
}

Masters of Science

in

Computer Engineering

Haibo Zeng, Chair

Patrick R. Schaumont

Cameron D. Patterson

April 27, 2018

Blacksburg, Virginia

Keywords: Simulink, Multicore, Software Synthesis, Partitioned scheduling Copyright 2018, Shamit Bansal 


\section{Optimal Implementation of Simulink Models on Multicore Architectures with Partitioned Fixed Priority Scheduling}

Shamit Bansal

(ABSTRACT)

Model-based design based on the Simulink modeling formalism and the associated toolchain has gained its popularity in the development of complex embedded control systems. However, the current research on software synthesis for Simulink models has a critical gap for providing a deterministic, semantics-preserving implementation on multicore architectures with partitioned fixed-priority scheduling. In this thesis, we propose to judiciously assign task offset, task priority, and task communication mechanism, to avoid simultaneous access to shared memory by tasks on different cores, to preserve the model semantics, and to optimize the control performance. We develop two approaches to solve the problem: (a) a mixed integer linear programming (MILP) formulation; and (b) a problem specific exact algorithm that may run several magnitudes faster than MILP. 


\section{Optimal Implementation of Simulink Models on Multicore Architectures with Partitioned Fixed Priority Scheduling}

Shamit Bansal

(GENERAL AUDIENCE ABSTRACT)

To save development time and money, automotive industries have been developing models using software, before implementing them directly on hardware. For reliability, the model generated from the software tool should behave in a well defined manner, coherent to the ideal design of the model. While the current tools are able to generate this reliable model for a single processor system, they are not able to do so for a system with multiple processors. When two or more processors contend to access the same resource at the same time, the existing tools are unable to provide a well defined execution order in their model. Since modern embedded systems need multiple processors to meet their increasing performance demands, it is imperative that the software tools scale up to multiple processors as well. In this work, we seek to bridge this gap by presenting two solutions that generate a deterministic software implementation of a system with multiple processors. In our solutions, we generate a model with well defined execution order by ensuring that at any given time, only one processor accesses a given resource. Furthermore, apart from ensuring determinism, we also improve upon the performance of the generated model by ensuring that there is minimal end-to-end latency in the system. 


\section{Dedication}

Dedicated to my parents 


\section{Acknowledgments}

First and foremost, I wish to thank my advisor Dr. Haibo Zeng for his guidance throughout

my masters. His consistent support and sound advice during the course of this research will remain as a solid foundation in my future professional career. I am grateful to him for always steering me in the right direction with his innovative ideas, great knowledge and experience. In the past year, he has not only helped me grow as a researcher but also as a professional with his mentorship. Lastly, I wish to thank him for being so patient with me, as without his support this thesis would not have been possible. I wish to thank the experts on my review committee Dr. Cameron Patterson and Dr. Patrick Schaumont for taking time out of their busy schedule to read this thesis and providing valuable feedback. I also wish to thank them for teaching me two of the best courses I have had the opportunity to attend at Virginia Tech. I am grateful to Yecheng Zhao for all his help during the course of this project. Being new to this domain, I was easily overwhelmed and confused in the beginning. But he patiently handled all my doubts and helped me understand the fundamental concepts of software programming as well as real-time systems. I will forever be indebted to my family back home for being the constant source of encouragement and support in my life. I wish to thank my labmate Prachi Joshi for being so helpful and providing valuable tips and suggestions on writing this thesis. I am thankful to my friend in Michigan, Uthara Menon for her support, motivation and advice on life in general. I would also like to thank all my friends in Blacksburg: Vamsi, Aarushi, Omkar, Tania, Kunal, Surabhi, Abhishek, Manish, Shruti, Akshay and Vibhav who made the last two years, the best years of my life. Last but not the least, I thank my childhood friend Shubham Sarin for always encouraging me and helping me stay confident and positive. 


\section{Contents}

List of Figures $\quad$ ix

List of Tables $\quad$ xi

1 Introduction $\quad 1$

1.1 Synchronous Reactive Models . . . . . . . . . . . . . . . . . 3

1.2 Software Synthesis of SR models . . . . . . . . . . . . . . . . 4

1.3 Our Contribution ........................... 7

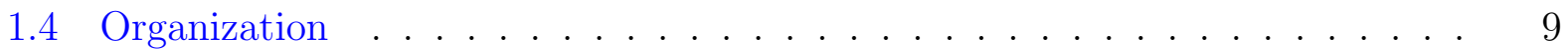

2 Related Work $\quad 10$

3 Mechanisms for Preserving Communication Semantics 14

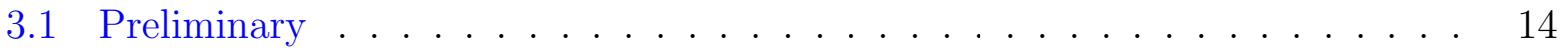

3.1.1 Semantics For Communication . . . . . . . . . . . . . . . 15

3.1.2 Challenges to Preserving Semantics . . . . . . . . . . . . . . 19

3.1.3 Preserving Semantics on Single-core . . . . . . . . . . . . . . 22

3.2 Task Model for Multicore Architecture . . . . . . . . . . . . . . . . . 26

3.3 Preserving Semantics on Multicore Architecture . . . . . . . . . . . . . 28 
3.3.1 Preserving Intra-core Communication Semantics . . . . . . . . . . . 28

3.3.2 Inter-core Communication Semantics . . . . . . . . . . . . . . . 31

4 Problem Formulation $\quad 35$

$\begin{array}{lll}5 & \text { MILP Formulation } & 37\end{array}$

6 Customized Optimization Algorithm 43

6.1 Problem Formulation for MIXO Framework . . . . . . . . . . . . 47

6.2 MIXO Computation . . . . . . . . . . . . . . . . . . . . 49

6.3 Feasibility Analysis for MIXO Based Framework . . . . . . . . . . . . 50

$6.3 .1 \quad$ Exact Feasibility Analysis . . . . . . . . . . . . . . . . 51

6.3 .2 Necessary Feasibility Analysis $1 \ldots \ldots$. . . . . . . . . . . 54

$6.3 .3 \quad$ Necessary Feasibility Analysis $2 \ldots \ldots \ldots$. . . . . . . . . 58

6.3.4 Final Algorithm for Feasibility Analysis . . . . . . . . . . . . 59

6.4 MILP for MIXO Based Framework . . . . . . . . . . . . . . . . . 61

6.5 Putting All Together - MIXO-Guided Framework . . . . . . . . . . . 61

$\begin{array}{llr}7 & \text { Results } & 66\end{array}$

7.1 Experiment on Random Systems _. . . . . . . . . . . . . . . 66

7.2 Fuel Injection System Case Study . . . . . . . . . . . . . . . . . 70

8 Conclusion and Future Work $\quad 72$ 
Bibliography 


\section{List of Figures}

1.1 Determinism in concurrent SR Models, such that black arrow represents trigger of task, dotted line represents global tick and the blue arrow represents data flow . . . . . . . . . . . . . . . . . .

1.2 Deterministic Execution Order in single-core, such that black arrow represents trigger of task and dotted line represents global tick . . . . . . . . . .

1.3 Deterministic Execution Order in multi-core, such that black arrow represents trigger of task and dotted line represents global tick . . . . . . . . . . .

3.1 Input/output relation with direct feedthrough on the communication link with (A) $T_{N_{i}}<T_{N_{j}}$ (B) $T_{N_{i}}>T_{N_{j}} \ldots \ldots \ldots \ldots$

3.2 Input/output relation with unit delay on the communication link with (A) $T_{N_{i}}<T_{N_{j}}(\mathrm{~B}) T_{N_{i}}>T_{N_{j}} \ldots \ldots \ldots \ldots$

3.3 Impact of Delay on Data Integrity on directfeedthrough with (A) $T_{N_{i}}<T_{N_{j}}$ (B) $T_{N_{i}}>T_{N_{j}} \ldots \ldots \ldots \ldots \ldots \ldots$

3.4 Impact of Delay on Data Integrity on a link with unit delay where (A) $T_{N_{i}}>$ $T_{N_{j}}(\mathrm{~B}) T_{N_{i}}<T_{N_{j}} \ldots \ldots \ldots \ldots \ldots$

3.5 Preserving Data Integrity by RT blocks for directfeedthrough in single-core with $(\mathrm{A}) T_{N_{i}}<T_{N_{j}}(\mathrm{~B}) T_{N_{i}}>T_{N_{j}} \ldots \ldots \ldots$

3.6 Preserving Data Integrity by RT blocks for unit delay link in single-core with (A) $T_{N_{i}}>T_{N_{j}}$ (B) $T_{N_{i}}<T_{N_{j}} \ldots \ldots \ldots \ldots \ldots$ 
3.7 Preserving the communication semantics for Intra-core directfeedthrough Link with (A) $T_{N_{i}}<T_{N_{j}}$ (B) $T_{N_{i}}>T_{N_{j}} \ldots \ldots \ldots \ldots \ldots \ldots \ldots \ldots \ldots \ldots \ldots$

3.8 Preserving the communication semantics for Intra-core unit-delay Link with (A) $T_{N_{i}}>T_{N_{j}}$ (B) $T_{N_{i}}<T_{N_{j}} \ldots \ldots \ldots \ldots \ldots$

3.9 Preserving the communication semantics for Inter-core directfeedthrough Link with (A) $T_{N_{i}}<T_{N_{j}}$ (B) $T_{N_{i}}>T_{N_{j}} \ldots \ldots \ldots \ldots$

3.10 Preserving the communication semantics for Inter-core Link with Unit-Delay with (A) $T_{N_{i}}>T_{N_{j}}$ (B) $T_{N_{i}}<T_{N_{j}} \ldots \ldots \ldots \ldots \ldots$

6.1 High Level Overview of Proposed Framework . . . . . . . . . . . . . . . . . 43

6.2 MUDA Framework Overview . . . . . . . . . . . . . . . . . . . . 52

6.3 MIXO-guided Framework . . . . . . . . . . . . . . . . . . . 60

7.1 Average Run-Time for 1000 Random Systems . . . . . . . . . . . . . 67

7.2 Average Run-Time for 50 tasks vs Utilization $\ldots \ldots$. . . . . . . . . 68

7.3 Normalized Objective Analysis for 1000 Random systems . . . . . . . . . . 69 


\section{List of Tables}

5.1 Notations Used in our implementation . . . . . . . . . . . . . . . 38

6.1 Example Test Case . . . . . . . . . . . . . . . . . . 45

7.1 Comparing performance of each Analysis . . . . . . . . . . . . . 68

7.2 Results for 1000 Random Systems . . . . . . . . . . . . . . . 70

7.3 Results for Fuel-Injection Case Study . . . . . . . . . . . . . . . . . 71 


\section{List of Abbreviations}

DAG Directed Acyclic Graph

DF Direct Feedthrough

ILP Integer Linear Programming

LH Low rate to High rate

MBD Model Based Design

MILP Mixed Integer Linear Programming

MIXO Minimal Infeasible partial eXecution Order

MUDA Maximal Unschedulable Deadline Assignment

RT Rate Transition

SR Synchronous Reactive

UD Unit Delay

WCET Worst Case Execution Time

WCRT Worst Case Response Time 


\section{Chapter 1}

\section{Introduction}

With the embedded systems becoming more complex day-by-day, the traditional manual software development is too slow and prone to errors. Thus the industries have shifted to using a model-based design (MBD) for developing embedded software for complex systems such as flight controllers, engine control and fuel injection systems. MBDs help shorten the development time by providing a visual abstraction of the system and allowing easier integration for complex systems. Additionally they allow the designer to evaluate the system performance, design trade-offs and even test the system functionality in a simulated environment. To provide maximum utility, the MBDs need to be accompanied by automatic code generation tools that can help in providing embedded software ready for deployment.

For the automotive industry, Simulink based MBDs with their well-defined formalism and associated toolchain have been a popular choice for a while now. For reduced implementation errors and faster turn-around times, tools such as Simulink Coder [45] are being used to automatically generate software implementations on single-core architectures. However, due to physical limitations the single-core architecture is reaching the limits of its computational capability. Thus, modern automotive systems are focusing on using multicore architecture to meet their increasing performance and efficiency demands. By allowing multiple processors to run concurrently, multicore architecture allows for a higher throughput than possible with a single processor. This migration however, creates a gap in research as the current solutions for semantics-preserving software implementation for Simulink models, including 
those provided by the commercial code generators, do not scale to multicore architectures. For example, the Simulink toolchain relies on users to specify the data communication mechanisms, and the generated coder may have non-deterministic behavior and cannot guarantee to be semantics preserving [12].

The necessary requirement of generating a reliable software implementation is that it should follow the semantics of the ideal model. With synchronous reactive (SR) as the underlying formalism, simulated Simulink models assume that the tasks have atomic operation that follow a defined causality order [41]. To implement SR models successfully for a multicore system, the challenge is to ensure that the generated implementation of the system follows the logical-time execution semantics. This is not a trivial problem, as in real-time implementation the blocks do not run with a zero-execution time but rather have an execution time dependent upon the scheduling policy as well as on the interference rising from contention for shared resource. As multiple tasks compete for the shared resources across multiple cores, the possible thread-interleavings may further give rise to multiple possible execution orders. Since, there is no defined execution order, the reliability of the generated software suffers by a great deal. For safety-critical embedded systems such as a flight management system, this non-determinism can prove to be quite fatal [20]. Furthermore, in order to ensure that the semantics of communication are preserved, the generated system sometimes requires addition of sample-and-hold buffers. However, these buffers may further add functional delay in the system delay blocks which adversely affects the end-to-end latency and may have critical impacts for control-command systems such as those used in avionics [22].

Our objective in this work is to ensure that the generated deterministic software implementation for multicore Simulink models preserves the logical-time execution semantics of a SR model while providing optimal control performance. 

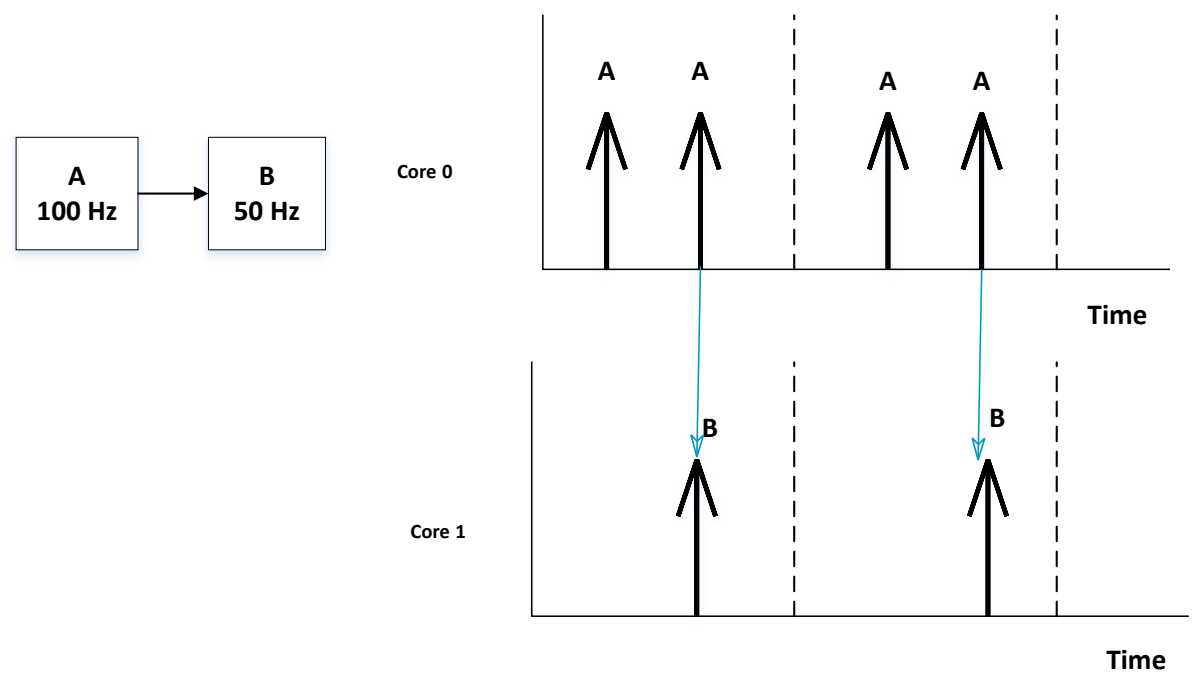

Figure 1.1: Determinism in concurrent SR Models, such that black arrow represents trigger of task, dotted line represents global tick and the blue arrow represents data flow

\subsection{Synchronous Reactive Models}

SR models can be viewed as logically timed models similar to the cycle-based systems present in hardware designs, where the concurrent actions of the system are associated with each tick of the global clock. This type of model ensures that for the same set of inputs, we get the same set of outputs at every tick. Since these models consider atomic operations, they do not allow multiple possible interleavings, thus providing a deterministic execution order in the system. This atomic operation can be seen in Figure 1.1, as A (producing output at $100 \mathrm{~Hz}$ ) and B (producing output at $50 \mathrm{~Hz}$ ) are triggered at the same time and B obtains the data from the most recent instance of A. The communication flow of the data represented by blue arrow shows this operation while assuming logical-time execution semantics. Since SR models assume atomic operation with negligible execution time, there is no data race condition and hence deterministic data transfer is ensured. The precision provided by SR models has further propagated its use in widespread industrial applications. Currently SR is the underlying modeling formalism for various languages such as Esterel[6], Lustre [24] and 
the Simulink graphical language[45]. The formal properties that these languages allow for an easier validation and verification of the generated model. For example, SCADE (Safety Critical Application Development Environment) is an industrial application of Lustre which is used in design of safety-critical flight controller software systems, engine control systems, automatic pilot systems, etc.

Thus if generated correctly, the software implementation based on SR formalism will always provide a deterministic execution order which is essential for any reliable implementation. In the following subsection, we briefly explain how we ensure that our generated model follows the logical-time execution semantics of the SR model for multicore systems.

\subsection{Software Synthesis of SR models}

When synthesizing software implementation of Simulink models, the generated implementation should preserve the semantics of the ideal model mentioned above. This is challenging as in real-time implementation the blocks do not run with a zero-execution time but rather have an execution time dependent upon the scheduling policy as well as on the interference rising from contention for shared resource. To ensure that the semantics of simulated model (as shown in Figure 1.1) are preserved in the generated single-core model, Simulink adds Rate Transition (RT) blocks as a form of wait-free buffer [45] between two communicating tasks (as shown in Figure 1.2). These blocks are required for ensuring data integrity as well deterministic transfer of data between tasks running at different rates $[12,50]$ (see Section 3.1.3). For a single-core architecture, using RT blocks with a defined priority assignment allows the generated software implementation to follow a deterministic execution order as shown in Figure 1.2. We see that by assigning A a higher priority than B and using RT block to buffer A's output, we can ensure the communication semantics of simulated model in Fig- 


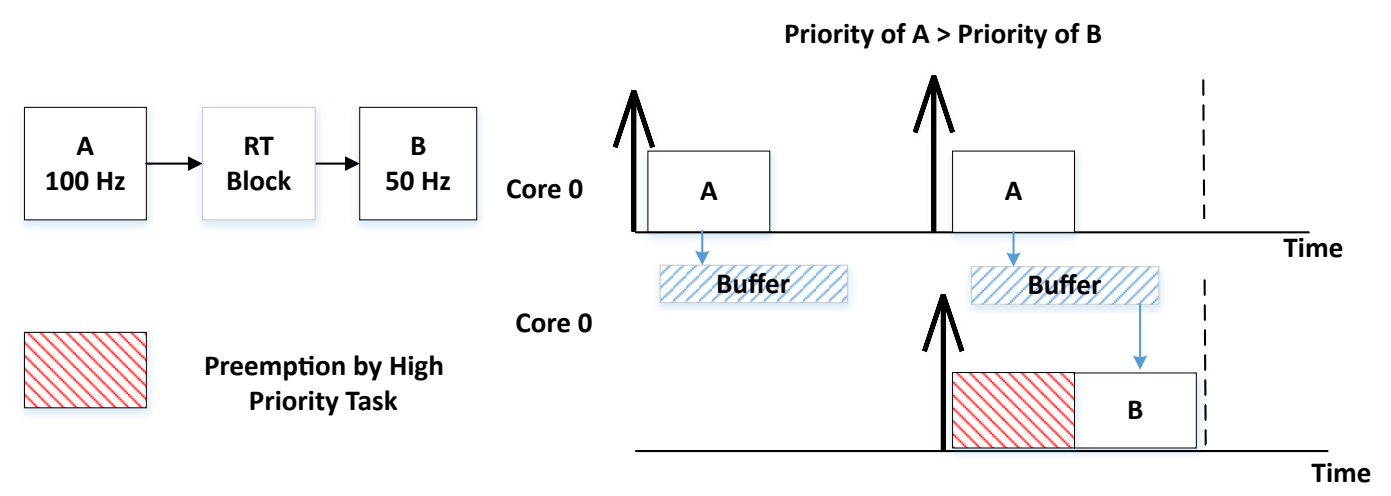

Figure 1.2: Deterministic Execution Order in single-core, such that black arrow represents trigger of task and dotted line represents global tick

ure 1.1 is preserved. Thus, even though the blocks execute in non-zero time, by assigning block A higher priority than $\mathrm{B}$ we ensure that when both $\mathrm{A}$ and $\mathrm{B}$ are triggered together, B is preempted by A. This preemption ensures that A updates the buffer before B can read it, thus allowing B to read the latest value produced by A.

However, in the case of multicore models with partitioned scheduling, the lack of global priority assignment prevents us from establishing a deterministic execution order, as the blocks on different cores are scheduled independently. As we can see from Figure 1.3(A), task B may have two possible execution windows (denoted by dotted box) based on the scheduling delay present in core 1 . This scheduling delay is dependent (1) upon the preemption of B by other high priority tasks in core 1 as well as (2) the interference rising from the contention for resources that are shared across multiple cores. These multiple execution orders not only result in an unpredictable timing model but may also violate the simulated communication semantics of Figure 1.1.

To avoid this pitfall, we use release times (offsets) in our implementation to enforce a global execution order to schedule the blocks on different cores. When A and B are triggered together as shown in Figure 1.3(B), releasing B only after A has completed its execution ensures a that B reads the latest value from the buffer as shown in Figure 1.1. This offset 


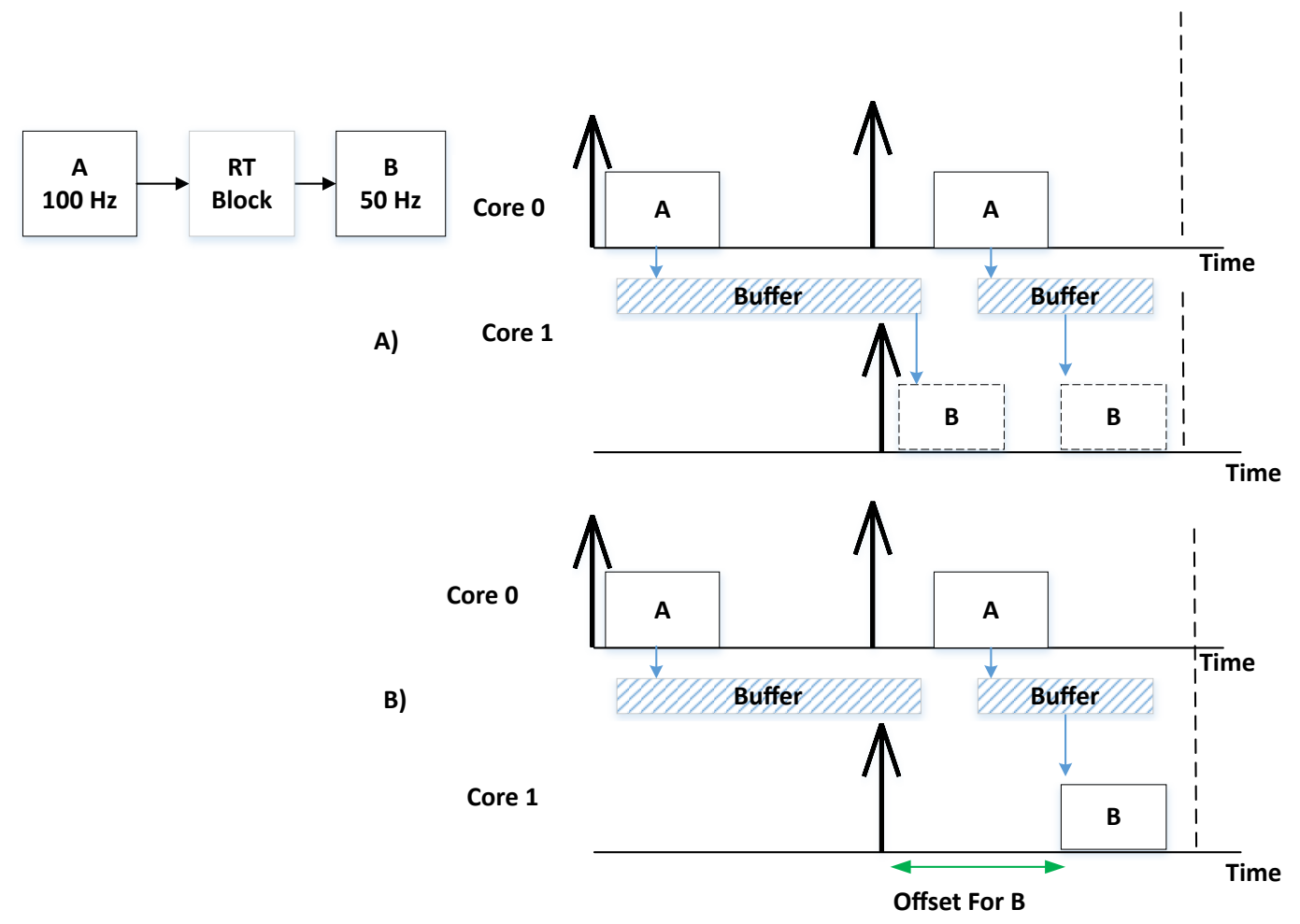

Figure 1.3: Deterministic Execution Order in multi-core, such that black arrow represents trigger of task and dotted line represents global tick

assignment ensures that a deterministic execution order is followed while ensuring data integrity. Furthermore, we also use this offset assignment to separate the execution windows of tasks that seek to access the same resource. Thus we need to ensure that when A is accessing the buffer, B should not be executing. In order to do so, we release B only when A is done with its execution and the buffer is free. This temporal isolation between communicating tasks further helps in timing predictability as now software on different cores does not access the same resource at the same time.

Combining separate execution windows with wait-free RT blocks will now allow the writer to write its data into a buffer and the reader to independently read from the given buffer index during its execution. This ensures data consistency and semantics preservation of the simulated model as shown in Figure 1.1. However, if the reader is unable to meet its 
deadline, the addition of this RT block sometimes requires using sample-and-hold operation to ensure data integrity (described in section 3.1.3). This sample-and-hold operation adds functional delay in the system by relaxing the input/output dependency within the same cycle (tick). While this relaxed dependency improves schedulability (see section 3.1.1), it can cause control performance degradation and even system instability [18].

The optimal software synthesis of multicore Simulink models can be formalized as the following design optimization problem: finding a deterministic implementation that follows the semantics of the ideal model and requires the addition of the minimum number of functional delays to do so. For this deterministic implementation, we need to ensure that all the tasks meet their respective deadlines (i.e. within each tick) while following the causality order of the simulated model. To solve this optimization problem, we briefly discuss our contribution in the following sub-section.

\subsection{Our Contribution}

In our work, we consider partitioned fix-priority scheduling due to its extensive use in industrial standards such as AUTOmotive Open Source ARchitecture (AUTOSAR), commercial real time operating systems such as VxWorks, and in particular, the code generators for Simulink such as Embedded Coder. Partitioned scheduling essentially means that no notion of global priority exists and the tasks cannot migrate between cores. Since there is no notion of global priority across cores, in order to ensure the deterministic implementation, we need to solve the system for the following

- assignment of release time of each task to ensure separate execution windows and establishing an execution order across all the cores 
- assignment of priorities to tasks on the same core, such that the tasks meet their deadlines and enforce an execution order within the core

- assignment of the communication mechanism i.e. addition of functional delays using sample-and-hold buffers, on communication links if required for preservation of communication semantics

The objective of our optimization problem is to find a deterministic implementation with minimal cost in terms of added delays for a given multicore system. In our analysis, we assume that the allocation of tasks to cores is already provided to us and leave the optimal partitioning scheme as future work.

In this thesis we provide two approaches to solve the optimal software synthesis problem. In the first approach we develop a Mixed Integer Linear Programming (MILP) formulation for the problem (see Chapter 5) that defines the model in terms of mathematical constraints and then searches for the optimal solution. Second, we develop a problem specific framework that starts with zero delay block assignment initially and then adds delay block to a link only if it is necessary for schedulability (see Chapter 6). The obtained results (see Section 7.1) show that our proposed framework is more scalable than the standard MILP while preserving the optimality of the solution, thus making it a suitable alternative for finding an optimal solution for medium and large sized systems.

For evaluating the performance of both the approaches, we perform our experiments on an industrial case study as well as on randomly generated systems. By evaluating the performance on a simplified version of the fuel injection systems [17], we show how efficient our proposed framework is when it comes to handling a real-world problem. The results from the case study show that our approach performs 2-3 orders of magnitude faster than the MILP while preserving the optimality of the solution. 


\subsection{Organization}

In this thesis, we organize the contents as following :

1. Chapter 2 discusses related work in this domain.

2. Chapter 3 discusses the semantics of the communication and the mechanisms used for preservation of the communication semantics.

3. Chapter 4 formally defines the optimization problem that we seek to solve.

4. Chapter 5 talks about the MILP formulation used to solve the defined optimization problem

5. Chapter 6 explains the problem specific exact algorithm that outperforms MILP

6. Chapter 7 discusses the results that we get from experiments on random systems as well as from the industrial case study for both the approaches

7. Chapter 8 provides the conclusion of this work along with future work that can be incorporated in this problem

\section{Conference}

The following is the conference to which this work has been submitted

- Bansal S., Zhao Y., Zeng H., Yang K. "Optimal Implementation of Simulink Models on Multicore Architectures with Partitioned Fixed Priority Scheduling", International Conference on Embedded Software (EMSOFT). Submitted. 


\section{Chapter 2}

\section{Related Work}

To provide a deterministic timing predictable model for multicore embedded systems, the interference from contention for shared resources has to be considered to estimate the execution time of each task [40]. A survey of this interference and its effect on response time has been done by Axer et al. [5], Wegener [48]. While computing the response time for multicore system is not our objective, it is relevant to discuss the common approaches that have been used to deal with the interference that rises when software on different cores access the same resource. The said approaches can be broadly divided into two categories as shown below:

$\triangleright$ Computing the impact of the interferences and adding them to analysis[3], [33]. Altmeyer et al. [3] in their work, provide a general framework used that analyzes the individual interferences on bus, memory as well as on the core to compute the net impact of interference on the response time. Rihani et al. [44] in their work build upon the generic framework developed in [3] to compute the impact of interference specifically for synchronous data flow graphs. Davis et al. [15] consider the memory demand and processor demand of each task and hence use it to compute the interference caused by the execution of a given task on the system, thus providing a more exact solution than [3]. Kang et al. [26] in their work compute the interference that rises from all 4 possible types of communication i.e. high priority to low priority on same core and different core as well as low priority to high priority on same core and different core. Our approach may be considered complimentary to all these approaches as we seek to minimize this interference for deterministic execution. Similar to our approach, 
Martinez et al. [32] in their work also develop a technique to reduce the contention by using slack-time and hence work with framework of [44] to show that reduced contention improves the performance. However, their work is not scalable and they left the optimization as future work. Kelter and Marwedel [27] analyze all possible paths of execution for a given task and thus provide the worst-case execution order. However, this technique is also not scalable to large systems and is currently implemented only for a single rate system with non preemptive execution, thus limiting its application. Thus we see that in order to obtain a deterministic execution order and save ourselves from state explosion, we need to mitigate the interference completely as shown below.

$\triangleright$ Mitigating the effects of such interferences by techniques such as temporal isolation [7] or resource partitioning [37]. The objective of this approach is to reduce the cost of interference on analysis of deterministic execution. Perret et al. [37] discuss how spatial isolation of resources and temporal isolation for execution (Time Division Multiplexed Arbitration for accessing the bus) can help in obtaining a deterministic multicore model. Maia et al. [30] provide this robust partitioning of resources by using isolated windows of execution for each task. Thus the execution of a task cannot have any impact on the execution of any other task. A real life application of these techniques have been presented in [20]. Durrieu et al. [20] have successfully developed a deterministic flight management system by using these isolated execution windows of the tasks. However, to manage the intra-core interference they impose non-preemptive scheduling whereas we account for that interference as well in our analysis. The work done by Carle et al. [8] also uses temporal isolation between dependent tasks to provide a deterministic multi core system. However, their schedulability analysis does not scale well and has a 1 hour-timeout rate for $50 \%$ cases at 50 tasks itself. Klikpo and Munier-Kordon [28] in their work develop a heuristic that uses temporal isolation for providing deterministic execution for a synchronous data flow graph. However, their work 
provides a sub-optimal solution and works only on a uniprocessor system. As mentioned in [34], synchronization of dependent tasks also has a cost on response time analysis. However, our approach successfully avoids this cost by using temporal isolation of dependent tasks, thus turning them into independent tasks.

Although our focus is on Simulink model, we discuss the related work in the broader context of Synchronous reactive (SR) model of computation, as it is the underlying modeling formalism for Simulink [45]. SR is supported in several other languages such as Esterel [6], Lustre [24], and Prelude [21, 35]. These synchronous languages have widespread industrial applications such as SCADE. As mentioned in Chapter 1, these languages are used for saftey-critical deterministic systems such as flight controller software systems, engine control systems etc. To propagate their use tools such as S2L [47] have been developed to translate a subset of Simulink to other synchronous languages.

On single-core platforms, Esterel or Lustre models are typically implemented as a single executable that runs according to an event server model [39]. The longest chain of reactions to any event shall be completed within the system base period (the greatest common divisor of all periods in the system). For multi-rate systems, this imposes a very strong condition on real-time schedulability that is typically infeasible in cost-sensitive application domains such as automotive [17]. The commercial code generators for Simulink models (such as Simulink Coder from MathWorks or TargetLink from dSPACE) provide two options. The first is a single-task (executing at the base period), which is essentially the same approach as [39]. The second is a fixed-priority multitask implementation, where one task is generated for each period in the model, and tasks are scheduled by Rate Monotonic policy. Caspi et al. [9] provide the conditions of semantics-preservation in a multi-task implementation. Di Natale et al. [16] propose to optimize the multitask implementation of multi-rate Simulink models with respect to the control performance and the required memory, and develop a branchand-bound algorithm. Later in [17], an ILP formulation is provided. For synchronous multi 
rate models Forget et al. [22] demonstrate how analysis of end-to-end latency is affected by such delays buffers that exists between tasks.

Comparably, the research on the implementation of SR models on multicore and distributed systems is rather limited. Prelude $[21,35]$ provides rules and operators for the selection of a mapping onto platforms with Earliest Deadline First (EDF) scheduling, including multicore architectures [36, 42]. The enforcement of the partial execution order required by the SR model semantics is obtained in Prelude by a deadline modification algorithm. The extension of the communication mechanisms including the RT block on multicore platforms is discussed in [49]. Pagetti et al. [36] provide a manual design experience for an avionics case study modeled in Simulink and implemented on a many-core platform. This case study is also used to develop a tool that generates code, where the tasks are time-triggered and the functional delays are presumed to be given [23]. Puffitsch et al. provide an approach to automatically map tasks to cores on a many-core architecture with EDF [42] or tick-based scheduling [43]. The commercial Simulink tool requires the user to specify if a delay block shall be added on each communication link and ensure the associated deadlines are met, but this is very difficult without automated tool support [45]. Overall, our work is the first to automate and optimize the synthesis of semantics-preserving software for Simulink models on multicore architectures with fixed-priority scheduling.

On distributed architectures, the implementation of SR models has been discussed in [10, 11, 38, 46]. Specifically, techniques for generating semantics-preserving implementations of SR models on Time-Triggered Architecture (TTA) are presented in [11]. The use of waitfree mechanisms, in particular the Simulink Rate Transition block to multicore platforms is discussed in [50], [25]. Methods for desynchronization in distributed implementations are discussed in $[10,38]$. A general mapping framework from SR models to unsynchronized architecture platforms is presented in [46], where the mapping uses intermediate layers with queues and then back-pressure communication channels. 


\section{Chapter 3}

\section{Mechanisms for Preserving Communication Semantics}

In this chapter, we formally present the problem of optimal software synthesis of Simulink models for multicore architecture. As mentioned in Chapter 1, our objective is to ensure that the generated implementation follows the logical-time execution semantics of the ideal model while providing optimal control performance. In this chapter we start with explaining the communication semantics of the ideal Simulink model. Once the communication semantics of the ideal model are established, we then proceed to discuss how the generated implementation can ensure the preservation of these communication semantics.

\subsection{Preliminary}

A Simulink model can be represented by a Directed Acyclic Graph (DAG) $\mathcal{G}=\{\mathcal{N}, \mathcal{E}\}$, where $\mathcal{N}=\left\{N_{1}, \ldots, N_{|\mathcal{N}|}\right\}$ is the set of nodes representing Simulink blocks. In this work, we will use the words terms node and block interchangeably for Simulink models. $\mathcal{E}=\left\{E_{1}, \ldots, E_{|\mathcal{E}|}\right\}$ is the set of edges representing the communication links between the blocks. In this thesis, we assume that each block is implemented by a dedicated task, and use the terms block and task interchangeably. This is consistent with the assumption made in similar works in $[2,35]$. Multiple Simulink blocks could be mapped to the same task running at a period equal to the 
greatest common divisor of these blocks' periods. The mapping of blocks to tasks presents another optimization problem and is left out as future work.

For synchronous model, we assume that within the model, the nodes are triggered periodically. We denote the period (inverse of the rate) of node $N_{i}$ as $T_{i}$. For each block $N_{i}$, we assign an activation offset (release time) $O_{i}$ that is smaller than its period $T_{i}$. Whenever $N_{i}$ is triggered, it will wait until $O_{i}$ time unit later to be ready for execution. Given $t \geq 0$, we define $n_{i}(t)$ to be the number of times that $N_{i}$ has been activated before or at $t$. Since blocks are triggered periodically, the $k$-th instance of $N_{i}$ is triggered at time $r_{i}(k)=k \cdot T_{i}$. Blocks interface with other blocks using a set of input ports and a set of output ports. Input ports carry signals sampled with the period $T_{i}$. The set of signals are produced with the same period on the output ports. Given $t \geq 0$, we define $n_{i}(t)$ to be the number of times that $N_{i}$ has been activated before or at time $t$. For Simulink models, we say that the input to the node is sampled with the period $T_{i}$. The input signals are processed by the node and the resulting output is a set of signals with the same period, produced on the output port of the same node.

\subsubsection{Semantics For Communication}

Each link $l_{i, j}=\left(N_{i}, N_{j}\right)$ in $\mathcal{E}$ connects the output port of node $N_{i}$ (the writer node) to an input port of node $N_{j}$ (the reader). In consistency with Simulink's assumption, we also assume that for each writer-reader relation, the periods of the reader and writer are harmonic i.e. the larger period is an integral multiple of the smaller period. If the output of $N_{j}$ is directly dependent on its input from $N_{i}$, then we say that $N_{j}$ consumes the data within the same tick as $N_{i}$, denoted by $N_{i} \rightarrow N_{j}$. We refer to this precedence as direct feedthrough dependency. This essentially means that $N_{j}$ depends upon the current instance of $N_{i}$. We can describe this relationship mathematically as shown in equation 3.1. Since we assume nodes 
are triggered periodically, let $N_{j}(k)$ denote the $k$-th instance of $N_{j}$. Furthermore, let $r_{j}(k)$ denote the time this instance is triggered, and $i_{j}(k)$ be its input. The SR semantics specify that $i_{j}(k)$ equals the output of the last occurrence of $N_{i}$, denoted by $o_{i}(m)$. The logicaltime semantics for SR models dictate that the output $o_{i}(m)$ should be triggered before the $k$-th occurrence of $N_{j}$. Thus the time at which $m$-th instance of the output update can be triggered latest is $r_{j}(k)$. By assuming that $m$-th instance is being triggered at $r_{j}(k)$ we can define the following equation for direct feedthrough:

$$
i_{j}(k)=o_{i}(m) \text {, where } m=\max \left\{n \mid r_{i}(n) \leq r_{j}(k)\right\} .
$$

Figure 3.1 illustrates a direct feedthrough relationship between a writer node $N_{i}$ and a reader node $N_{j}$. The x-axis represents time. In the figure, to ensure direct feedthrough (i.e. to ensure that the reader reads the most recent value) (a) if $T_{N_{i}}<T_{N_{j}}$ we have $i_{j}(k)=o_{i}(m)$ and $i_{j}(k+1)=o_{i}(m+2)$, (b) if $T_{N_{i}}>T_{N_{j}}$, we have $i_{j}(k)=i_{j}(k+1)=o_{i}(m)$ and $i_{j}(k+2)=i_{j}(k+3)=o_{i}(m+1)$

The SR semantics also allow for delayed communication, where the delay added is limited to one unit in Simulink (the more general case of multiple delays are discussed in works such as [31], [22]). If the communication is delayed, $N_{j}$ does not depend on the output of the most recent activation of $N_{i}$; instead it now reads the previous value. We denote this by $N_{i} \stackrel{-1}{\rightarrow} N_{j}$. In this case, we say that the $k$-th instance of reader reads from $(m-1)$-th instance of writer (from the previous tick), where $m$-th instance is the most recent writer instance that is triggered before $k$-th instance of reader as shown in equation 3.2

$$
i_{j}(k)=o_{i}(m-1), \text { where } m=\max \left\{n \mid r_{i}(n) \leq r_{j}(k)\right\}
$$

Figure 3.2 shows the effect of adding a unit delay on the communication link. In the 

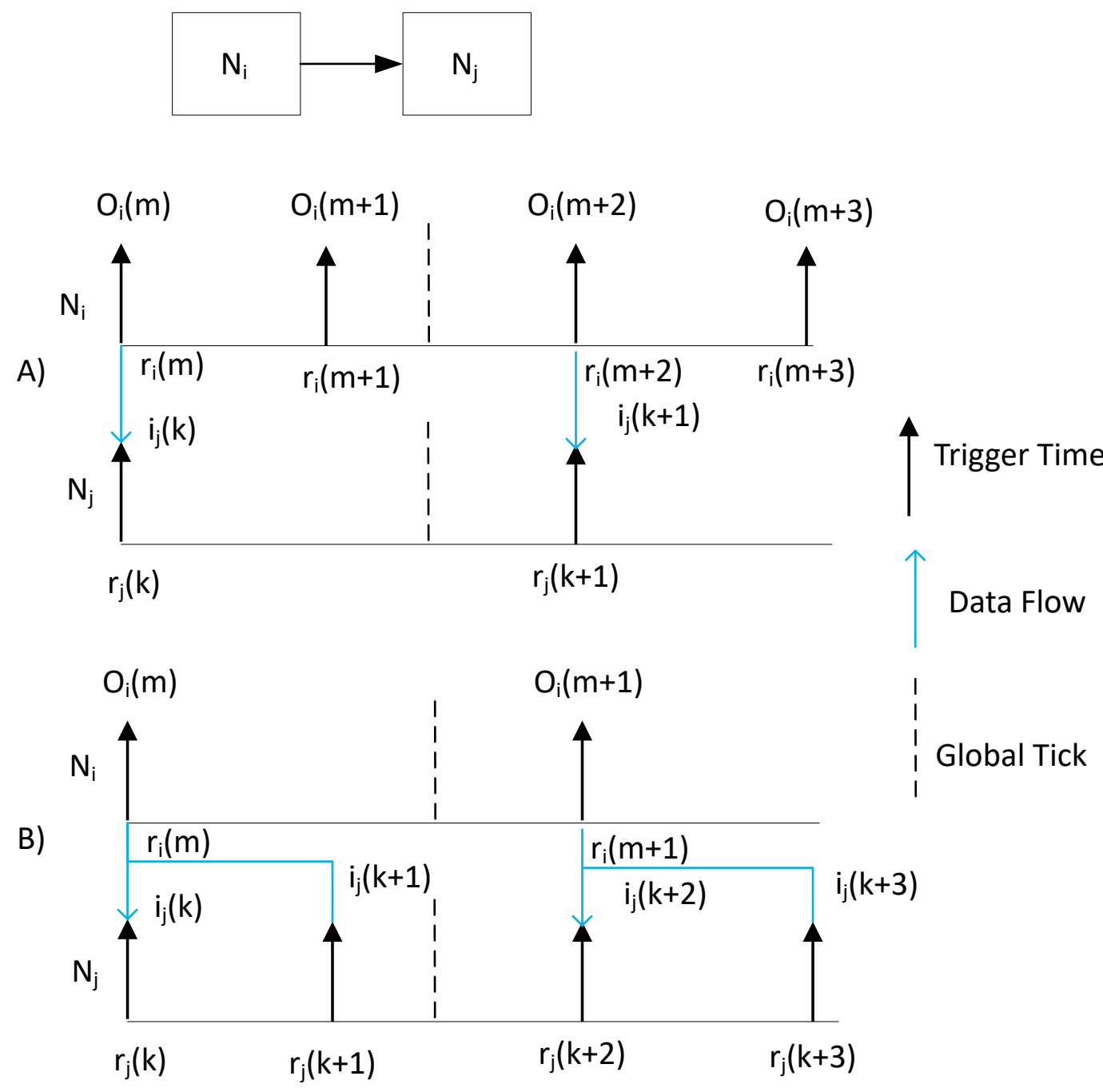

Figure 3.1: Input/output relation with direct feedthrough on the communication link with (A) $T_{N_{i}}<T_{N_{j}}(\mathrm{~B}) T_{N_{i}}>T_{N_{j}}$

figure, to ensure unit delay (i.e. to ensure that the reader reads the previous value) (a) if $T_{N_{i}}<T_{N_{j}}$ we have $i_{j}(k+1)=o_{i}(m+1)$ and similarly $i_{j}(k)=o_{i}(m-1)$, (b) if $T_{N_{i}}>T_{N_{j}}$, we have $i_{j}(k+2)=i_{j}(k+3)=o_{i}(m)$ and similarly $i_{j}(k)=i_{j}(k+1)=o_{i}(m-1)$. We can see that there is more time between the instance at which the output is produced and the instance at which it is consumed. In this case, the reader does not have to finish its computation within the same tick thus relaxing the input/output dependency within each tick of execution. This provides some flexibility in implementing schedulability in the system. 

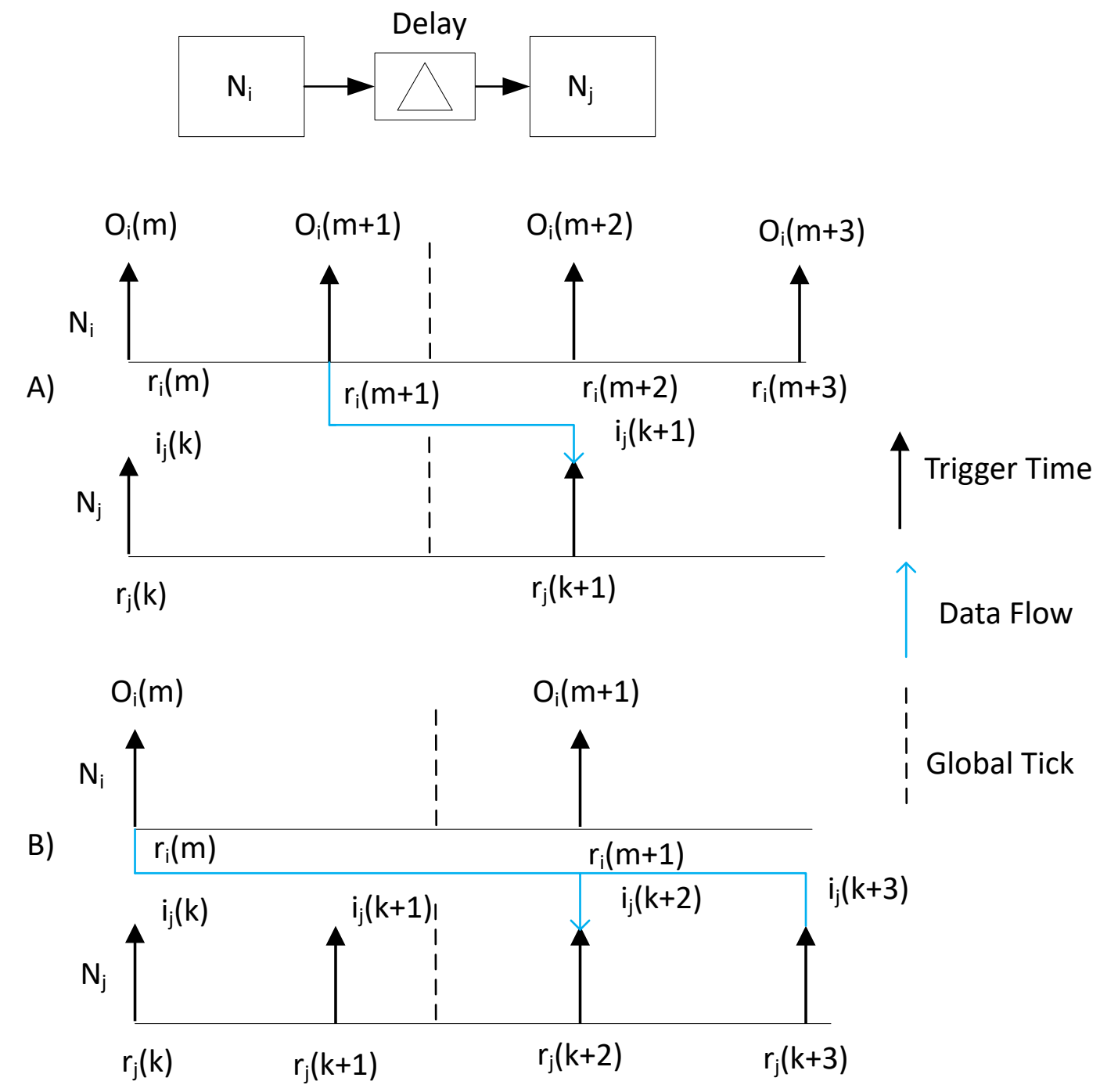

Figure 3.2: Input/output relation with unit delay on the communication link with (A) $T_{N_{i}}<T_{N_{j}}(\mathrm{~B}) T_{N_{i}}>T_{N_{j}}$

However, this delay requires additional storage in memory for buffering, as the data remains in the buffer till the next tick when it is finally consumed by the reader. Furthermore, the added delay increases end-to-end latency which might cause performance degradation especially for control algorithms [18], [22]. For safety-critical embedded systems such as flight management system [20], increasing this end-to-end latency beyond a particular value will result in an unstable operation. Thus, we need to minimize the unit delay block addition 
in a given system to ensure optimal control performance.

In summary, in SR semantics the data exchanged by two communicating blocks must be clearly defined by the model. With direct feedthrough dependencies, the reader reads the data produced by the most recent occurrence of the writer. With delayed communication, data from the previous instance is used. In both cases however, there should be no confusion and the producer of each data item consumed by a reader is explicitly defined by the model and the computation of reader and writer should complete before the next tick.

A cyclic dependency is possible if $N_{i}$ and $N_{j}$ (directly or indirectly) depend on each other in a feedthrough dependency. This results in a fixed point problem and can violate determinism in SR semantics by making the output dependent on scheduling. Simulink simply disallows such cyclic dependencies. In this work, following the approach in Simulink, we assume that the system does not have any cyclic dependencies, hence we work only with Directed Acyclic Graphs in our analysis.

\subsubsection{Challenges to Preserving Semantics}

When generating software code for Simulink functional models, we must ensure that the implementation behaves identically to the simulated model. An additional complication here is that it is relatively easier for the simulation engine to preserve the model semantics since the engine controls virtual time and blocks are assumed to execute in zero virtual time. However, in reality blocks take time to execute, and preemptions and scheduling delays may cause differences between the simulated and implemented signal flows. In addition to the aforementioned factors, the interference from contention for shared resources may further add an additional delay to the execution of a block.

While considering these delays, to ensure data integrity, we seek to implement a schedulable 
system that follows the logical-time execution semantics as shown in Figure 3.1 and Figure 3.2. In the following sub-section we discuss how these delays may result in violation of communication semantics for the generated model.

\section{Impact of Execution Delay on Data Integrity}

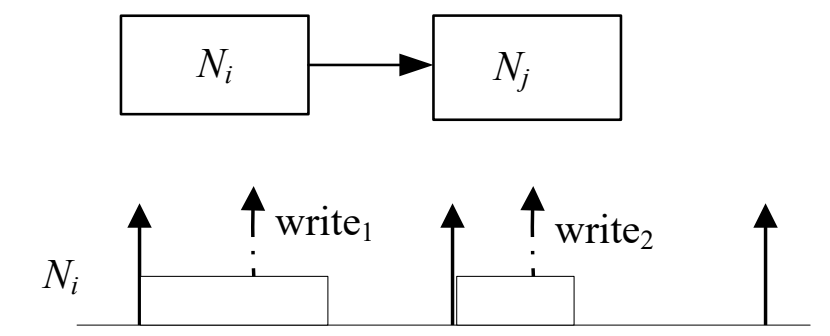

A)
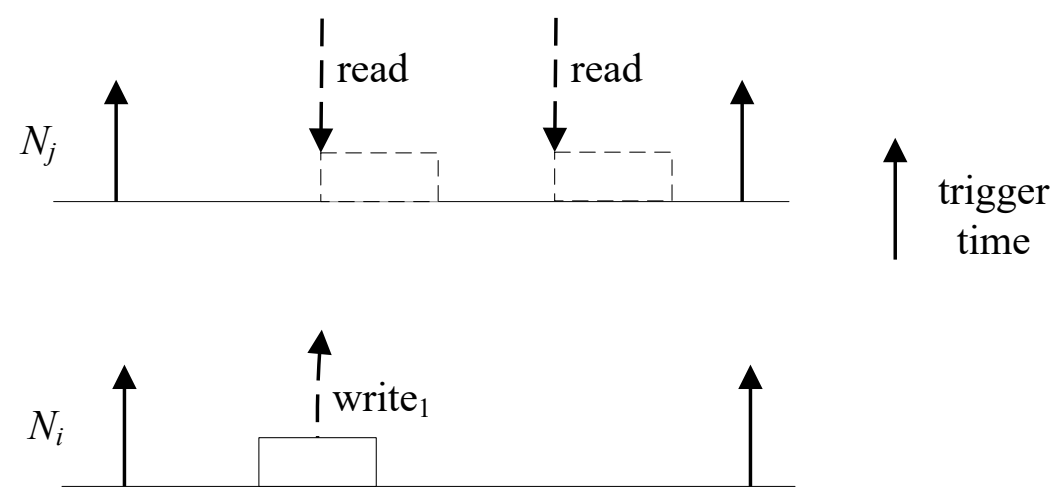

B)

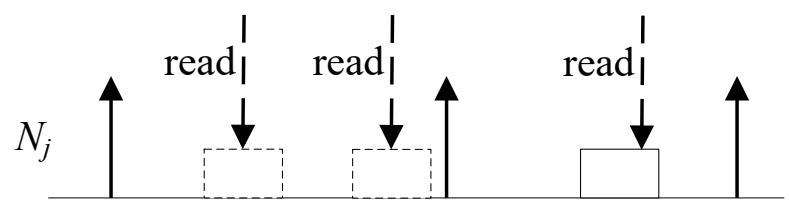

Figure 3.3: Impact of Delay on Data Integrity on directfeedthrough with (A) $T_{N_{i}}<T_{N_{j}}$ (B) $T_{N_{i}}>T_{N_{j}}$

Considering the semantics defined in section 3.1.1, we see that the scheduling delays may violate the data integrity for both cases as following :

$\triangleright$ Case 1: The reader depends upon the most recent instance of the writer when triggered, as shown in Figure 3.3. In this case the implementation should follow the semantics of direct- 
feedthrough (as shown in Figure 3.1). We use the dotted blocks to show how the scheduling delay may result in possible execution windows of the reader. In this figure, we have (A) a high-rate writer communicating to a low-rate reader and (B) a low-rate writer communicating to a high-rate reader. For Figure 3.3(A), based upon when the reader starts executing, the data read by the reader may come from the first instance (write $)_{1}$ ) or the second instance of the writer $\left(\right.$ write $\left._{2}\right)$. However, the simulated model semantics from equation (3.1) dictate that the reader should always read the value provided by the most recent instance of writer (write $)$. Similarly, for Figure 3.3(B), the possible execution windows of the first instance of the reader may result in loss its data integrity.

$\triangleright$ Case 2: The reader task reads the previous value of the writer instance when triggered,
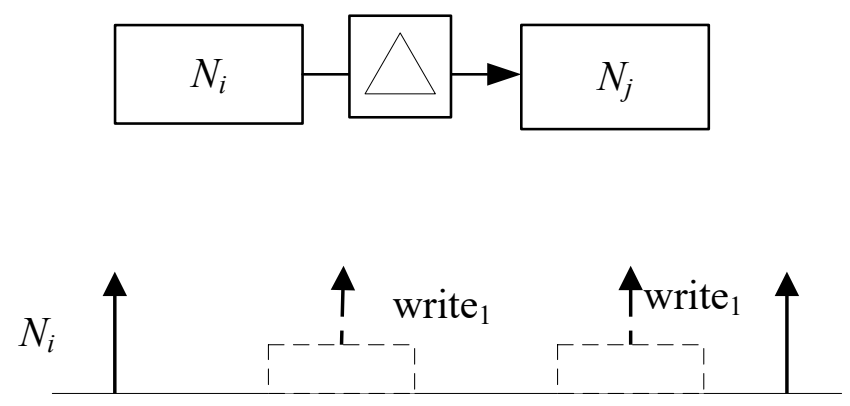

A)

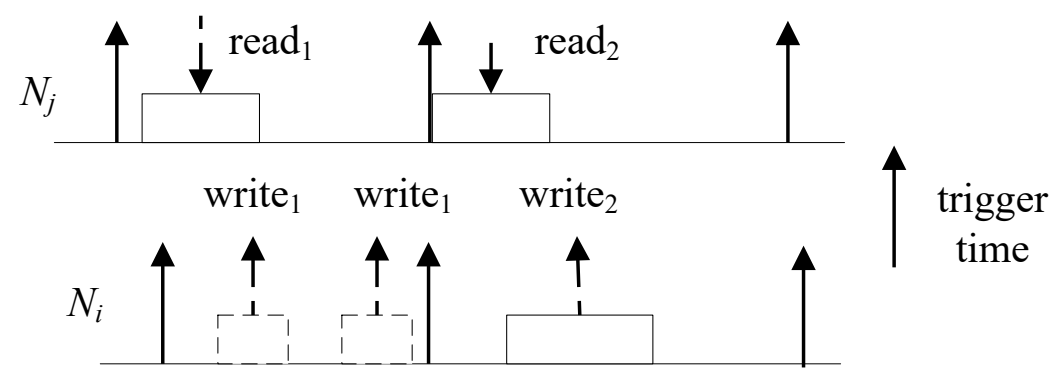

B)

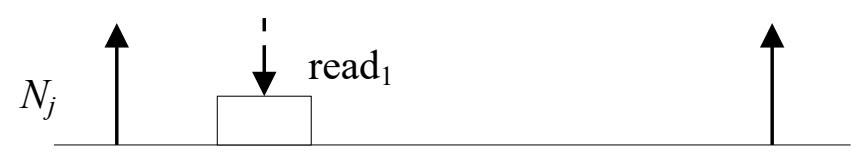

Figure 3.4: Impact of Delay on Data Integrity on a link with unit delay where (A) $T_{N_{i}}>T_{N_{j}}$ (B) $T_{N_{i}}<T_{N_{j}}$ 
as shown in Figure 3.4. In this case the implementation should follow the semantics of unit delay (as shown in Figure 3.2). We represent the possible executions of the writer task with dotted lines. In this figure, we have (A) a low-rate writer communicating to a high-rate reader (B) a high-rate writer writing to a low-rate reader. Thus for Figure 3.4 (A), we can see that based upon the release time, the input to the second reader $\left(\operatorname{read}_{2}\right)$ may be the same as read $_{1}$ or an updated value by the writer $\left(\right.$ write $\left._{1}\right)$. However, the simulated model semantics from equation (3.2) dictate that the input to the reader can only be updated by the next instance of writer (occurring in the next cycle/tick). Thus within this cycle both the readers should have same input i.e. read $_{1}$ and read $_{2}$ should be same. A functional delay in this case is clearly needed to allow that the reader gets the input from the previous instance

of the writer, along with an initial input for the first instance of the reader task. Similarly for Figure $3.4(\mathrm{~B})$, the reader should execute before the first instance of the writer $\left(\right.$ write $\left._{1}\right)$ and correspondingly second instance of writer (write $_{2}$ ).

We see that for a single-core architecture, Simulink provides a solution to both these problems as shown below.

\subsubsection{Preserving Semantics on Single-core}

Simulink solves both of the above mentioned problems as well as ensures data consistency for single-core using a mechanism known as Rate Transition (RT) blocks [45] (as shown in Figure 3.5 and Figure 3.6). RT blocks are a special implementation of wait-free methods. These blocks placed between the writer and the reader (as shown in Figure 3.5 and Figure 3.6), forward appropriate data from the writer to the reader, and provide initial data values when necessary. RT blocks are only applicable to one-to-one communication. However, one-to- $n$ communication can still be implemented using RT buffers as $n$ separate one-to-one links. Furthermore, RT blocks are a restricted version of wait-free methods. Compared to 
the generic wait-free methods [13], they require that the sender and receiver have harmonic periods. Harmonic periods ensure that the larger period is a multiple of the smaller period. Also, for our analysis we call the larger period to be the hyperperiod. Thus, we need to ensure that within a given hyperperiod, events happen in the same causality order as the simulated model.

The RT block comprises of two functions : output update function (denoted by striped box in our figures) and a state update function (denoted by gridded box in our figures). If we consider RT block as a buffer, then state update function can be considered as writing the data into the buffer. Similarly, output update function can be considered as reading the data from the buffer. Now we see how this RT block can be used to ensure data integrity for both cases mentioned in previous section.

$\triangleright$ Case 1: For the first case mentioned above in Figure 3.3, the RT block needs to behave like a Zero-Order Hold block as shown in Figure 3.5. Thus RT block buffers the data from the writer till the next instance of the reader is activated. The RT block's output update function (shown by striped box) executes at the rate of the slower block (at the rate of reader for Figure $3.5(\mathrm{~A})$ and at the rate of writer for Figure $3.5(\mathrm{~B}))$ but within the writer block, thus ensuring that the input to the reader is not updated till the next instance of slower block is triggered. Also, the state update (shown by gridded box) occurs within the task and at the priority of the writer block, thus ensuring that the output by each instance of writer is stored in the buffer. If the writer instance executes before the corresponding reader in the hyperperiod, we refer to RT blocks associated with this execution order as direct feedthrough $(D F)$.

$\triangleright$ Case 2: For the second case mentioned above in Figure 3.4, the RT block needs to behave like a Unit Delay block as shown in Figure 3.6. Thus RT block should provide an initial value in the start and buffer the output from the writer till the next instance of writer is triggered. The RT block state update function (shown by gridded box) should execute in 

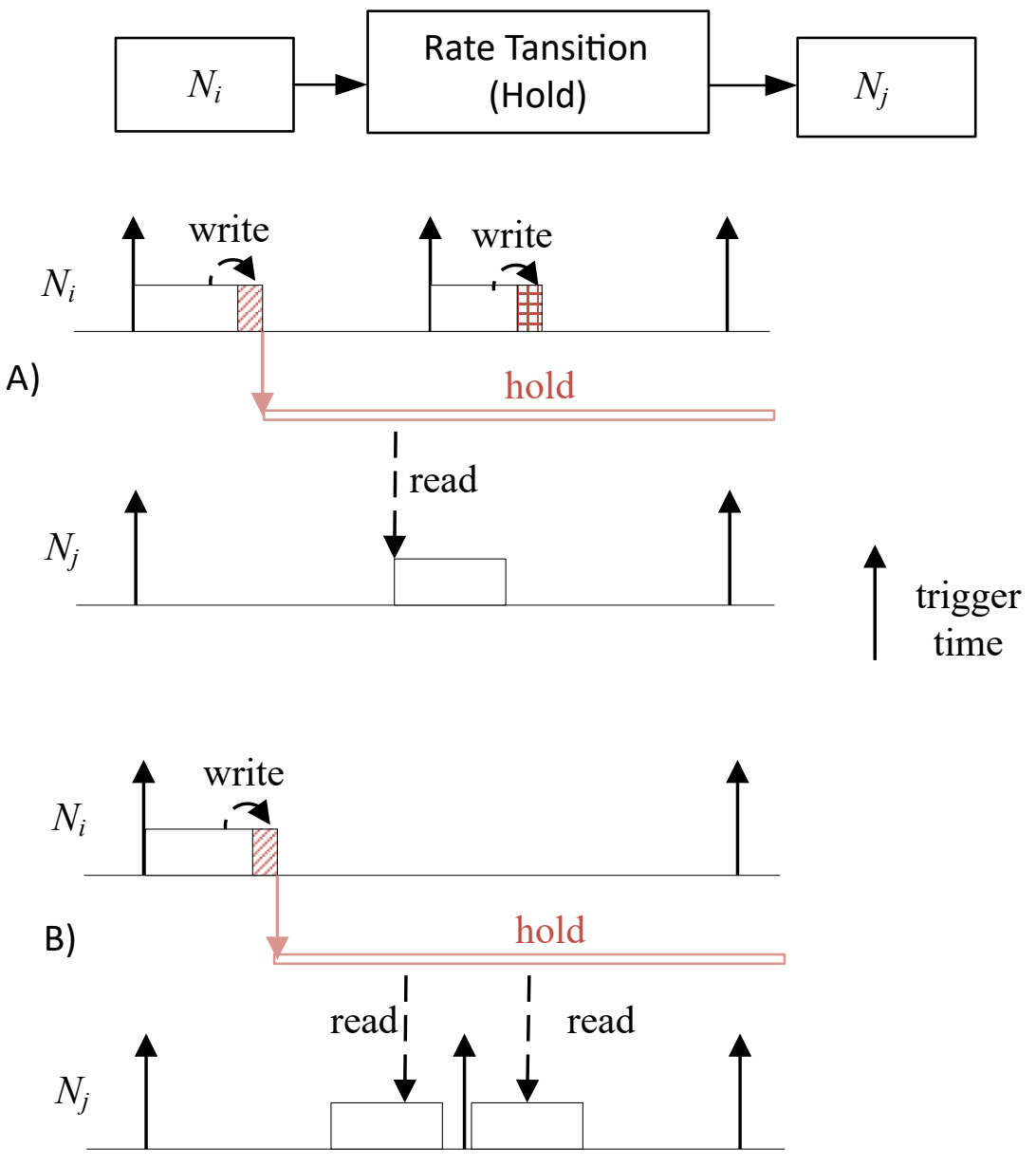

Figure 3.5: Preserving Data Integrity by RT blocks for directfeedthrough in single-core with (A) $T_{N_{i}}<T_{N_{j}}(\mathrm{~B}) T_{N_{i}}>T_{N_{j}}$

the context of the writer task, thus ensuring that the output of each writer instance is stored in the buffer. The RT block output update function should run in the context of the reader block, but at the rate of the slower block (at the rate of writer for Figure $3.5(\mathrm{~A})$ and at the rate of reader for Figure 3.5 (B)). This will ensure that the reader blocks read the same value, provided by the previous instance of the writer. For the case when the reader executes before the writer when triggered together, we refer to RT blocks associated with this execution order as Unit Delay (UD). This type of RT blocks results in additional functional delay equal to the writer's period as the data produced by the writer will be consumed in the next period. This causes an adverse effect on the end-to-end latency thus eventually 

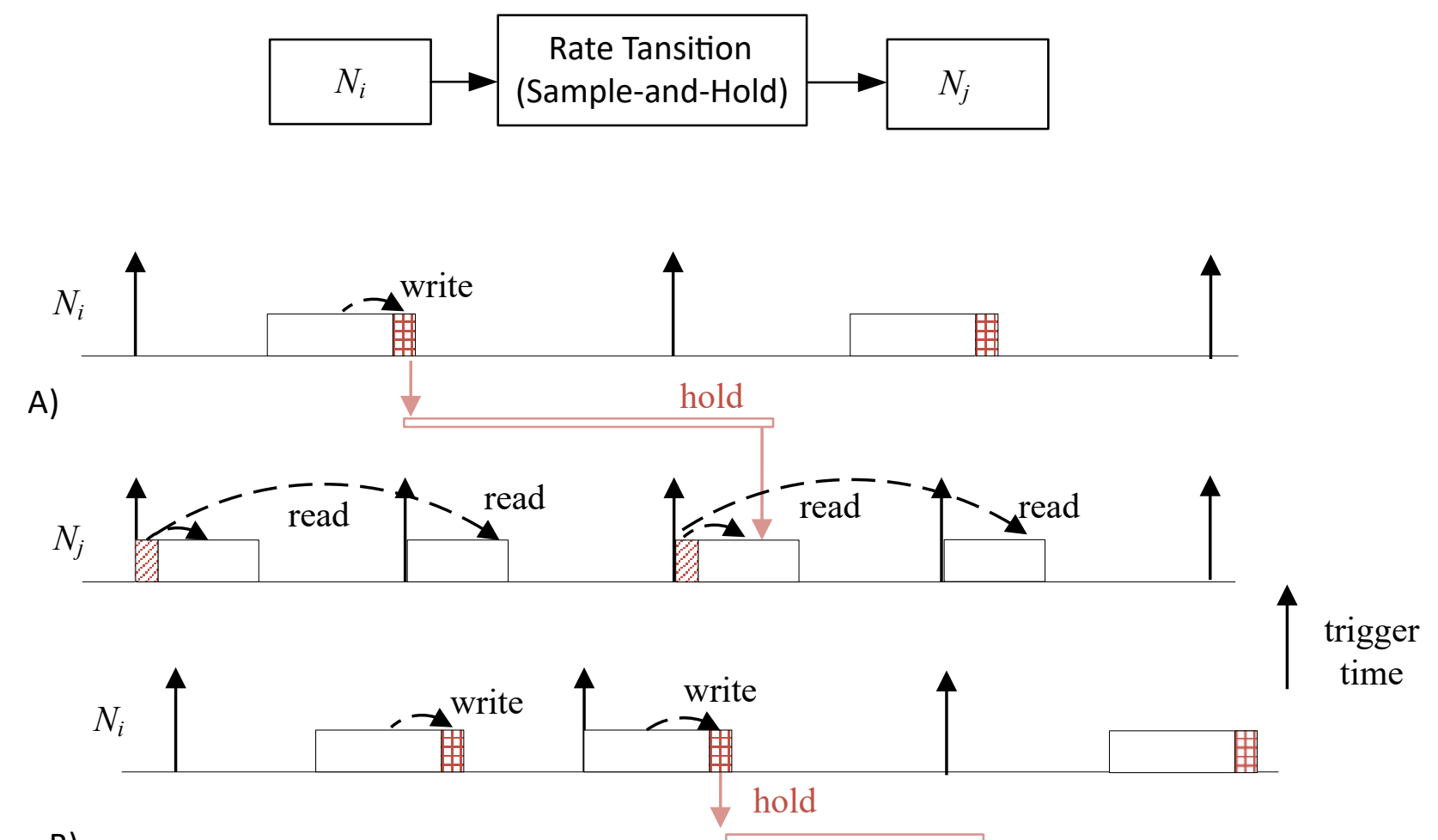

B)

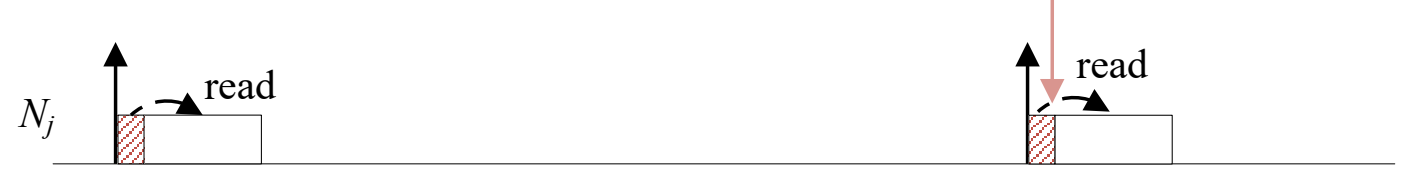

Figure 3.6: Preserving Data Integrity by RT blocks for unit delay link in single-core with (A) $T_{N_{i}}>T_{N_{j}}(\mathrm{~B}) T_{N_{i}}<T_{N_{j}}$

degrading the performance of the system [49].

With the above discussion, we see how the Simulink uses RT blocks to preserve the communication semantics on a single-core architecture. The problem becomes more complex for partitioned multicore architecture as there is no notion of global priority. Hence, in order to establish an execution order in the multicore architecture we use release time of each task. In the following section, we first discuss the task model that will be used in our analysis. Using that task model, we then proceed to explain how this execution order based on release times can be used to preserve the semantics for multicore architecture. 


\subsection{Task Model for Multicore Architecture}

We focus here on multitask implementations since they are much more efficient [18] and allow us to fully utilize a multicore architecture. In multitask implementation of Simulink models, blocks are mapped to fixed-priority tasks or threads scheduled by an RTOS. In our work we assume that the core allocation for each task is given to us as an input. We leave this partitioning scheme as a part of future work.

A task $\tau_{i}$ implementing node $N_{i}$ is characterized by the following parameters: $C_{i}$, denoting the task's Worst Case Execution Time (WCET) free from all interferences; $T_{i}$, the task's period which is the same as the period of $N_{i} ; D_{i}=T_{i}$, the deadline of task i.e. the amount of time a task has from its trigger time to the time instant at which it must finish its execution; $E_{i}$, the core allocated to the task $\tau_{i} ; p_{i}$, the task's priority; $\alpha_{i}$, the set of tasks that write to task $\tau_{i} ; \beta_{i}$, the set of tasks that read from $\tau_{i}$;

In addition, we use synchronized triggering of tasks, and introduce the offset (release time) $O_{i}$, which is the difference between the trigger time and the activation of the task instance. For our analysis, we define response time $R_{i}$ of task $\tau_{i}$ as the time difference between when the task finishes execution and the the time it is activated. The communication link between a writer task $\tau_{i}$ and reader task $\tau_{j}$ is denoted as $l_{i, j}$. Furthermore, we denote the presence of unit delay RT block on a communication link as $D B_{i, j} \forall l_{i, j}$. We define the response time of the output update function running in the context of reader $\tau_{i}$ as $R_{R T i}$ (discussed in section 3.1.3). Further notations are present in Table 5.1 in Chapter 5.

For a schedulable system we calculate the response time for a task and check if the task finishes before its deadline. We do this by considering both inter core as well as intra core interference :

- Intra Core Interference: The delay to the execution of task $\tau_{i}$ rising from all the tasks 
that preempt the execution of task $\tau_{i}$. We denote $h p(i)$ as the set of tasks that belong on the same core as $\tau_{i}$ and have a higher priority than $\tau_{i}$. Thus for execution window of $R_{i}$, task $\tau_{i}$ will be preempted at least $\frac{R_{i}}{T_{j}} \forall j \in h p(i)$. Preemption of $\tau_{i}$ by $\tau_{j}$ will result an addition of $\frac{R_{i}}{T_{j}} \cdot C_{j}$ to the execution of $\tau_{i}$. Thus the computed net interference is given as $\sum_{j \in h p(i)}\left\lceil\frac{R_{i}}{T_{j}}\right\rceil \cdot C_{j}$.

- Inter Core Interference: By using RT blocks and separating the execution windows of the communicating tasks, we remove the inter-core interference completely from calculation of response time.

Thus we end up with the calculation of Response Time of the task $\tau_{i}$ as shown in equation $(3.3)$

$$
R_{i}=C_{i}+\sum_{j \in h p(i)}\left\lceil\frac{R_{i}}{T_{j}}\right\rceil \cdot C_{j}
$$

where $h p(i)$ is the set of tasks that have higher priority than $\tau_{i}$ and are allocated to the same core. As seen from section 3.1.3 we also need response time of the output update function to ensure that for the case of unit delay, the writer can execute only after the output update function is completed. For output update function of RT block $R T_{i}$ running in the context of the reader task $\tau_{i}$, we assume the Worst Case Execution Time $\left(C_{R T}\right)$ to be negligible and the priority of the output update function to be the same as that of the reader task. By following the same procedure as calculation of $R_{i}$, we compute the response time of output update function as shown in equation 3.4

$$
R_{R T i}=C_{R T}+\sum_{j \in h p(i)}\left\lceil\frac{R_{R T i}}{T_{j}}\right\rceil \cdot C_{j} \text { s.t. } C_{R T} \approx 0
$$

Thus essentially the output update function has a response time equal to the net interference 
occurring due to preemption of task $\tau_{i}$ by other tasks running on the same core. Now that we have defined the task model and the semantics of communication, we move towards discussing how the communication semantics for this task model are preserved.

\subsection{Preserving Semantics on Multicore Architecture}

In partitioned multicore architectures, we need to ensure that the generated execution order follows the simulated causality order while preserving the communication semantics as done for single-core in Figure 3.5 and Figure 3.6. To do so, we need to make sure that the partial execution order between any two communicating blocks $N_{i}$ and $N_{j}$ follows the semantics between the writer and reader as shown in Figure 3.1 and Figure 3.2.

Definition 3.1. We define a partial execution order $\boldsymbol{f}_{\boldsymbol{i}, \boldsymbol{j}}$ in our system to denote that block $N_{i}$ executes before block $N_{j}$ whenever they are triggered together.

Example 3.2. In Figure 3.5, we say that the partial execution order between $N_{i}$ and $N_{j}$ is $f_{i, j}$, as $N_{i}$ executes before $N_{j}$ in the hyperperiod. similarly, for Figure 3.6, we say that the partial execution order between $N_{i}$ and $N_{j}$ is $f_{j, i}$.

In this section, we use definition 3.1 to formally define how the partial execution order should be used to preserve the communication semantics for both inter-core and intra-core communication links.

\subsubsection{Preserving Intra-core Communication Semantics}

When the reader and writer are assigned to the same core, we have a well-defined priority order to determine the execution order of the generated model. Thus the execution order in 
this case should follow the semantics as defined for single-core in Figure 3.5 and Figure 3.6. $\triangleright$ Case 1: The reader instance is directly dependent upon the output from the current writer instance. Thus the execution of the writer block $N_{i}$ is followed by the execution of the corresponding reader instance $N_{j}$, as shown in Figure 3.7. This partial execution order should ensure that the system follows the the semantics of Figure 3.1. For this case, the RT block should behave like a directfeedthrough RT block. Thus the state update function is executed within the writer task and the output update function (striped box) is executed at the rate of the slower block. To ensure that partial execution order $f_{i, j}$ follows the semantics of equation (3.1), we (a) assign block $N_{i}$ higher priority than block $N_{j}$ and (b) activate $N_{i}$ before $N_{j}$. If we assume the activation time of block $N_{i}$ is $O_{i}$ and the priority is $p_{i}$, we say that:

Principle 1. For a given intra-core communication link from $N_{i}$ to $N_{j}$, execution order $f_{i, j}$ enforces the following constraint to imply the use of a directfeedthrough RT block:

$$
O_{i} \leq O_{j} \bigwedge p_{i}>p_{j}
$$

$\triangleright$ Case 2: The reader instance depends upon the previous instance of the writer. Thus, reader $N_{j}$ executes before writer block $N_{i}$ when triggered together, as shown in Figure 3.8. The semantics for this execution order should follow the semantics for a relaxed dependency as shown in Figure 3.2. For this case, the RT block behaves like a Unit Delay block plus a Hold block (Sample and Hold). Thus the state update function (gridded box) executes within the writer block and the output update function (striped box) executes in context of reader at the rate of the slower block. To ensure that partial execution order $f_{j, i}$ follows the semantics of equation (3.2) we (a) assign block $N_{j}$ higher priority than block $N_{i}$ and (b) 


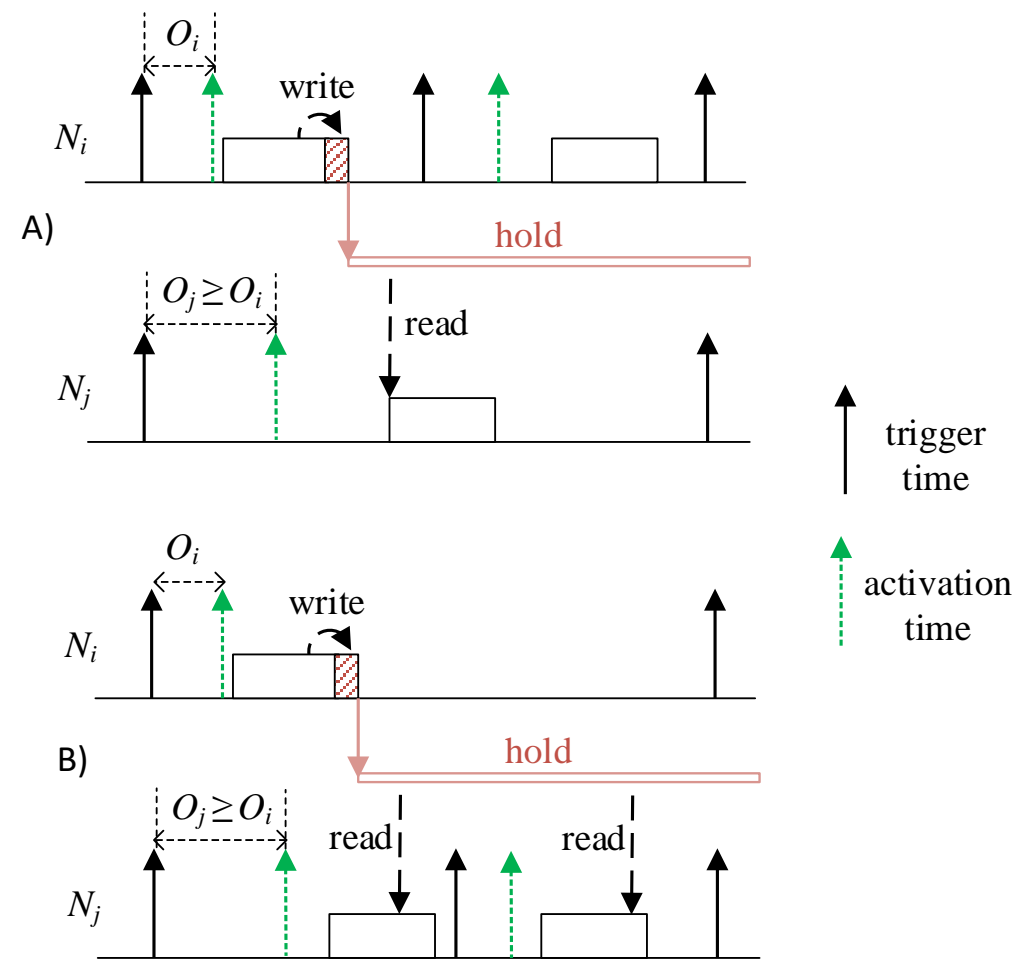

Figure 3.7: Preserving the communication semantics for Intra-core directfeedthrough Link with (A) $T_{N_{i}}<T_{N_{j}}$ (B) $T_{N_{i}}>T_{N_{j}}$

activate the output update function before $N_{i}$. If we assume the activation time of block $N_{i}$ is $O_{i}$ and the priority is $p_{i}$, we say that

Principle 2. For an intra-core communication link from $N_{i}$ to $N_{j}, f_{j, i}$ implies the use of a unit delay $R T$ block and enforces the following constraint:

$$
O_{j} \leq O_{i} \bigwedge p_{i}<p_{j}
$$

Now, we proceed to see how this semantics-preserving execution order can be defined for inter-core communication links. 


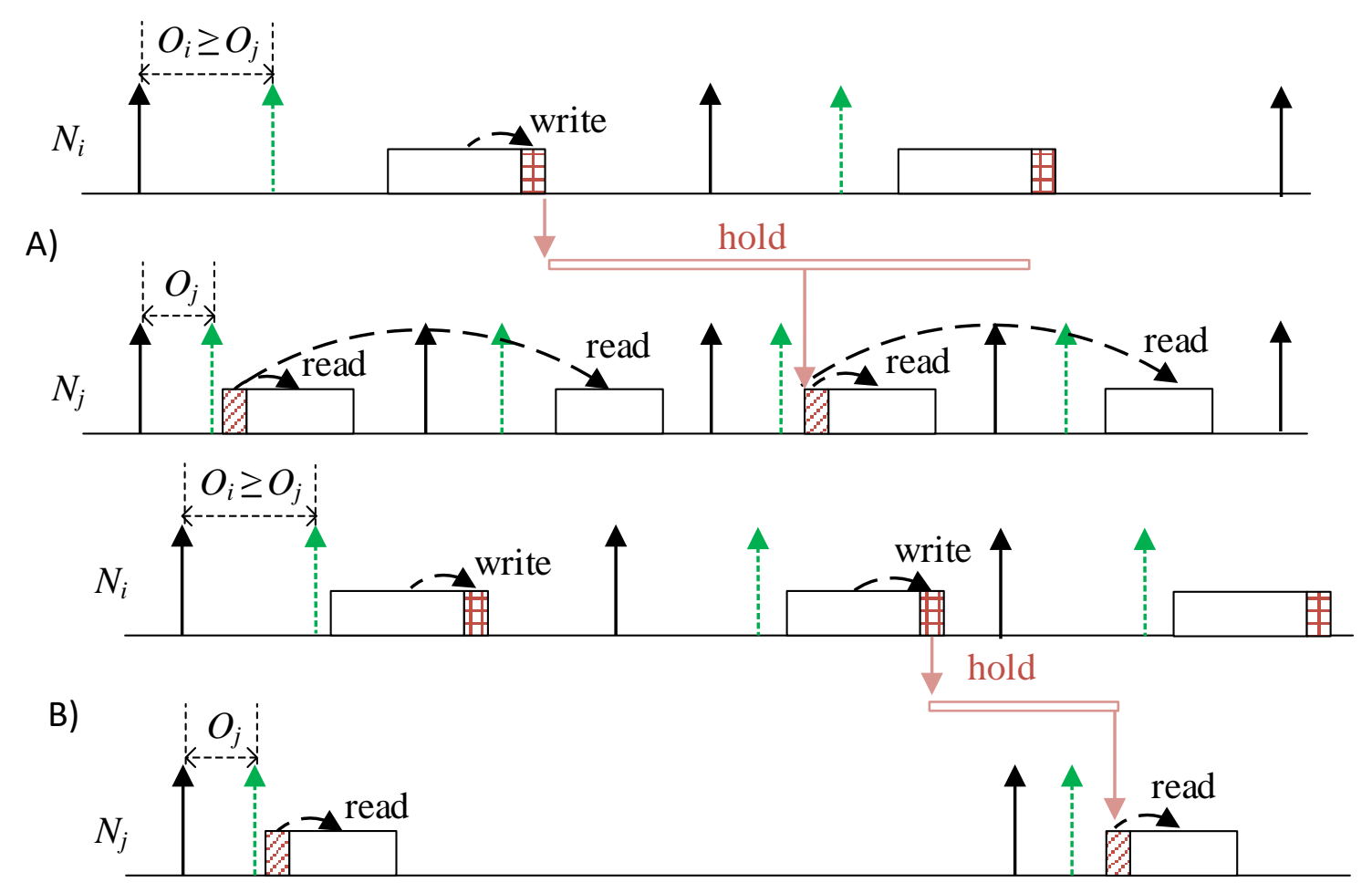

Figure 3.8: Preserving the communication semantics for Intra-core unit-delay Link with (A) $T_{N_{i}}>T_{N_{j}}(\mathrm{~B}) T_{N_{i}}<T_{N_{j}}$

\subsubsection{Inter-core Communication Semantics}

When the reader and writer blocks are assigned to different cores with partitioned scheduling, preserving the SR semantics is more challenging since there is no notion of global priority. We consider a mechanism that combines the RT block and offset assignment, the later assign a release offset to blocks to separate the execution windows of the communicating blocks on different cores and enforce a global execution order. The behavior of RT block in multicore is the same as that for the single-core as shown in Figure 3.5 and Figure 3.6.

$\triangleright$ Case 1: The reader instance directly depends upon the current writer instance. Thus, the writer block $N_{i}$ executes before the corresponding reader $N_{j}$, and we use a directfeedthrough 
RT block as shown in Figure 3.9. This execution order follows the semantics of Figure 3.1 such that the output update function (striped box) runs within the writer block at the rate of the slower block. For inter-core link, the partial order $f_{i, j}$ can preserve the semantics of equation (3.1) by activating the reader $N_{j}$ with an offset $O_{j}$ that should be no smaller than the sum of the worst case response time $R_{i}$ and the offset $O_{i}$ of the writer block $N_{i}$. This ensures that the state update function is executed and the buffer holds the latest value before the reader is activated. If we assume the activation time of block $N_{i}$ is $O_{i}$ and the response time is $R_{i}$, we say that:

Principle 3. For any inter-core communication link from $N_{i}$ to $N_{j}, f_{i, j}$ implies the use of a directfeedthrough $R T$ block and enforces the following constraint:

$$
R_{i}+O_{i} \leq O_{j}
$$

$\triangleright$ Case 2: The input to the reader depends upon the previous instance of the writer. This execution order should follow the semantics of Figure 3.2. Thus, the writer block $N_{i}$ starts executing after the output update function (striped box) is executed, as shown in Figure 3.10. Hence we need to ensure that the state update function (gridded box) cannot update the RT block before the reader has the chance to read it, as shown in Figure 3.6. We can ensure $f_{j, i}$ preserves the semantics of equation (3.2) by activating the writer $N_{i}$ with an offset $O_{i}$ no smaller than the offset $O_{j}$ of the reader $N_{j}$ plus the worst case response time $R_{R T j}$ of the output update function of the RT block (executing in the context of $N_{j}$ ). If we assume the activation time of block $N_{j}$ is $O_{j}$ and the response time of the output update function (in the context of reader $N_{j}$ ) is $R_{R T j}$, we say that 


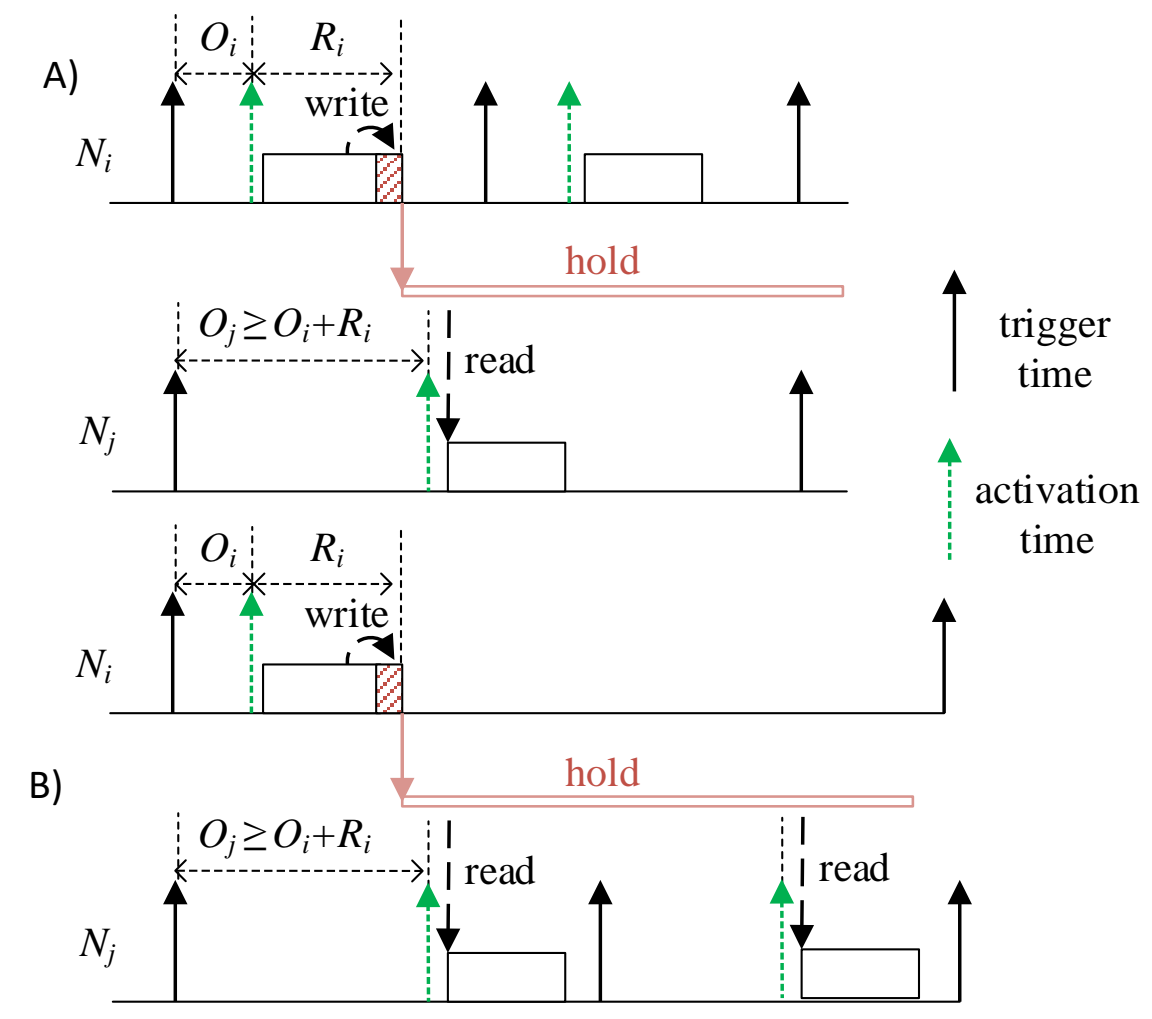

Figure 3.9: Preserving the communication semantics for Inter-core directfeedthrough Link with (A) $T_{N_{i}}<T_{N_{j}}$ (B) $T_{N_{i}}>T_{N_{j}}$

Principle 4. For any inter-core communication link from $N_{i}$ to $N_{j}, f_{j, i}$ implies the use of a unit delay RT block and enforces the following constraint:

$$
R_{R T j}+O_{j} \leq O_{i}
$$

With the above methods to synchronize the activations of the writer and the reader, we ensure that the obtained execution order is following the model semantics for both types of behavior (feedthrough and unit delay RT blocks). Additionally, for a schedulable execution order each block should finish its execution $\left(O_{i}+R_{i}\right)$ before its deadline $\left(D_{i}=T_{i}\right)$ which 


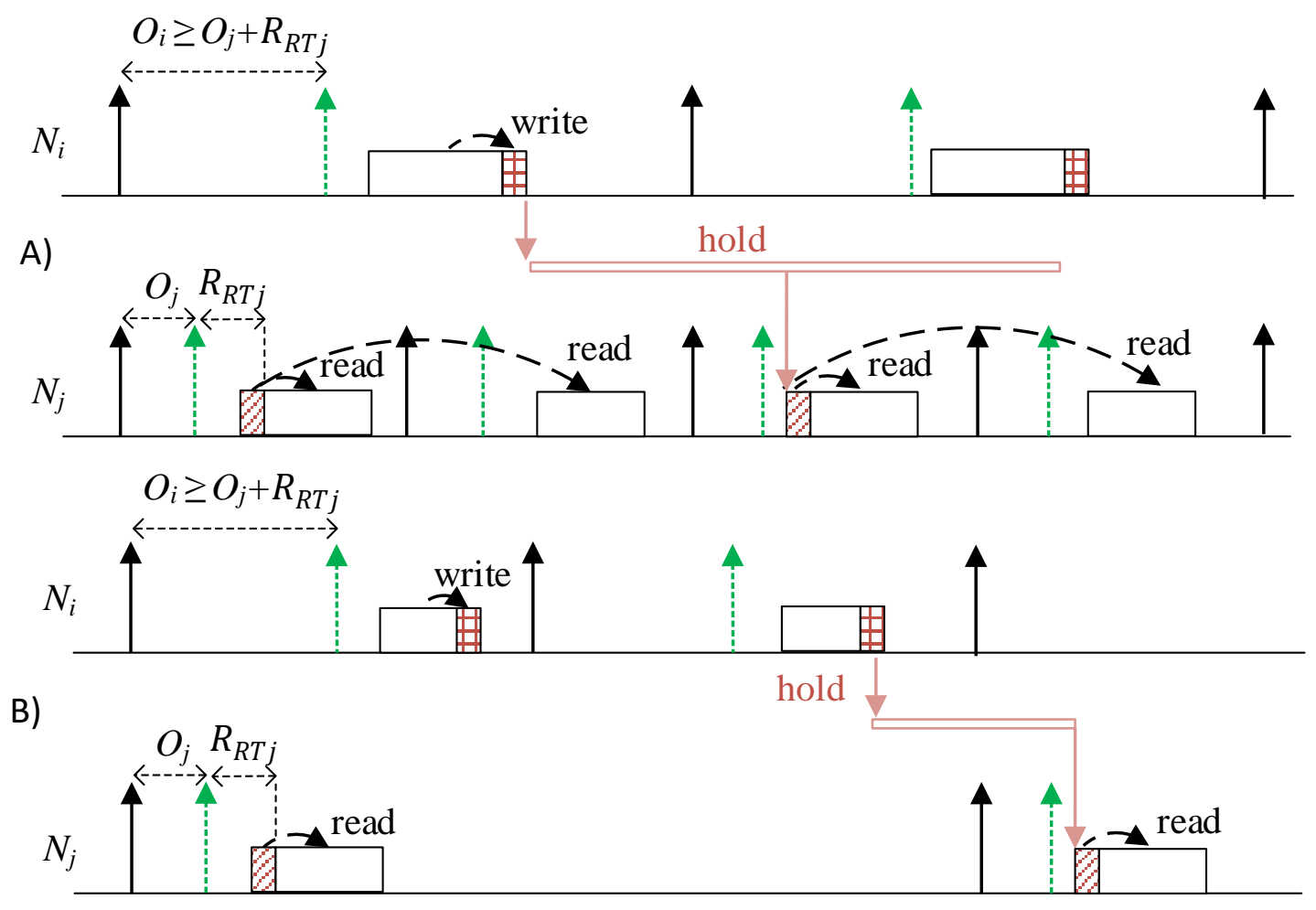

Figure 3.10: Preserving the communication semantics for Inter-core Link with Unit-Delay with (A) $T_{N_{i}}>T_{N_{j}}$ (B) $T_{N_{i}}<T_{N_{j}}$

adds another constraint for each block.

$$
R_{i}+O_{i} \leq T_{i}, \forall i
$$

Thus for a reliable software synthesis, we see that the generated execution order should preserve the communication semantics for multicore architecture for both feedthrough and unit delay RT blocks while ensuring schedulability. Furthermore, we need to reduce the number of unit delay RT blocks in the system for optimal performance. In the following chapter, we proceed to formulate the problem to see how the generated implementation can provide optimal performance while preserving the semantics for simulated model. 


\section{Chapter 4}

\section{Problem Formulation}

Now that the semantics for communication have been defined, we can now focus on formally defining the optimization problem for a given system (introduced in Chapter 1). In this work, we are interested in providing a generated implementation that follows the semantics of the simulated model and adds minimal unit delay in the system. As seen from equations (3.5), (3.6), (3.7) and (3.8) computing this execution order further involves: assigning priorities to tasks, assigning offsets to all tasks, and assigning delays to communication links. The design constraint is to ensure schedulability on all cores by meeting the deadlines of all tasks (as shown in equation (3.9)). The objective is to minimize the unit delay block count added by the execution order while ensuring schedulability.

For a link $\tau_{i} \rightarrow \tau_{j}$, we use the principles 1, 2, 3, 4 (defined in section 3.3) to say that

- partial execution order $f_{i, j}$ implies the use of direct-feedthrough RT block i.e. $D B_{i, j}=$ 0 . Additionally, to ensure that the generated model follows the logical-time execution semantics, design constraints (3.5), (3.7) and (3.9) should be satisfied

- partial execution order $f_{j, i}$ implies the use of a unit-delay RT block i.e. $D B_{i, j}=1$. Additionally, to ensure that the generated model follows the logical-time execution semantics, design constraints (3.6), (3.8) and (3.9) should be satisfied

Thus our optimal software synthesis should find an execution order that follows the communication semantics of the simulated model and adds minimal functional delay in the system. 
To represent this problem in a mathematical form, we introduce a helper binary variable $\boldsymbol{t}_{\boldsymbol{i}, \boldsymbol{j}}$ defined as

$$
t_{i, j}= \begin{cases}1, & f_{i, j} \text { is enforced } \\ 0, & \text { otherwise }\end{cases}
$$

Each link $l_{i, j}$ introduces two binary variables $t_{i, j}$ and $t_{j, i}$ corresponding to two possible partial execution orders. The optimization problem can then be formally expressed as

$$
\min _{\forall \mathbf{P}, \mathbf{O}, \mathbf{t}} \sum_{\forall l_{i, j}} w_{i, j} \cdot t_{j, i}
$$

s.t. $\mathbf{P}, \mathbf{O}$ satisfy the implied design contraint by $f_{i, j}, \forall l_{i, j}$

$$
\begin{aligned}
& R_{i}+O_{i} \leq D_{i}, \forall i \\
& t_{i, j}+t_{j, i}=1, \quad \forall i \neq j \\
& t_{i, j} \geq t_{i, k}+t_{k, j}-1, \quad \forall i \neq j \neq k
\end{aligned}
$$

where $\mathbf{P}=\left[p_{1}, \ldots p_{n}\right]$ and $\mathbf{O}=\left[O_{1}, \ldots O_{n}\right]$ represent the vectors of priority and offset assignment respectively, $\mathbf{t}=\left[t_{i, j}, t_{j, i} \mid l_{i, j}\right]$ is the set of execution order variables, and $w_{i, j}$ is the cost on adding a functional delay to the link $l_{i, j}$. Thus formulation (4.2) seeks to find the minimal cost of unit delay addition for all possible priority orders, offset assignment and execution orders while ensuring the schedulability of the system. The last two sets of constraints correspond to anti-symmetry and transitivity of the partial execution orders and are used to ensure that a valid execution order exists. The former means that if $\tau_{i}$ has a higher order than $\tau_{j}\left(t_{i, j}=1\right)$, then $\tau_{j}$ must have a lower order than $\tau_{i}\left(t_{j, i}=0\right)$. The later enforces that if $\tau_{i}$ has a higher order than $\tau_{k}\left(t_{i, k}=1\right)$ and $\tau_{k}$ has a higher order than $\tau_{j}\left(t_{k, j}=1\right)$, then $\tau_{i}$ must have a higher order than $\tau_{j}\left(t_{i, j}=1\right)$.In the following chapter, we discuss the proposed solutions that we use to solve the optimal software synthesis problem for the defined system model. 


\section{Chapter 5}

\section{MILP Formulation}

In this chapter, we discuss the standard MILP formulation that solve the optimization problem described in Section 4. We discuss a Mixed Integer Linear Programming (MILP) formulation that essentially converts the model into a set of mathematical equations and then solves those equations in accordance with a defined objective. To find the optimal solution, MILP uses branch and cut algorithm and applies cuts at every node to remove infeasible solutions till it finally reaches an optimal solution. However, this method of analysis makes it unscalable, as for larger systems the model becomes too huge and the branch and cut algorithm takes too much time to find the optimal solution. To get the solution quickly for large systems we then introduce a more efficient framework which finds the same optimal solution as MILP but has a much less run-time as compared to MILP (shown in Chapter 6). Within this MILP formulation, we will use notations present in Table 5.1. As mentioned in Section 4, the problem for finding the optimal execution order involves

- assigning priorities to tasks such that constraints (3.5), (3.6) are satisfied

- assigning offset and response time assignment such that constraints (3.5), (3.6), (3.7), (3.8) and (3.9) are satisfied

- ensuring minimal unit delay RT block count

After defining all the constraints and the objective function in terms of mathematical equations, we use IBM ILOG CPLEX Optimization studio [1] to solve the model. 


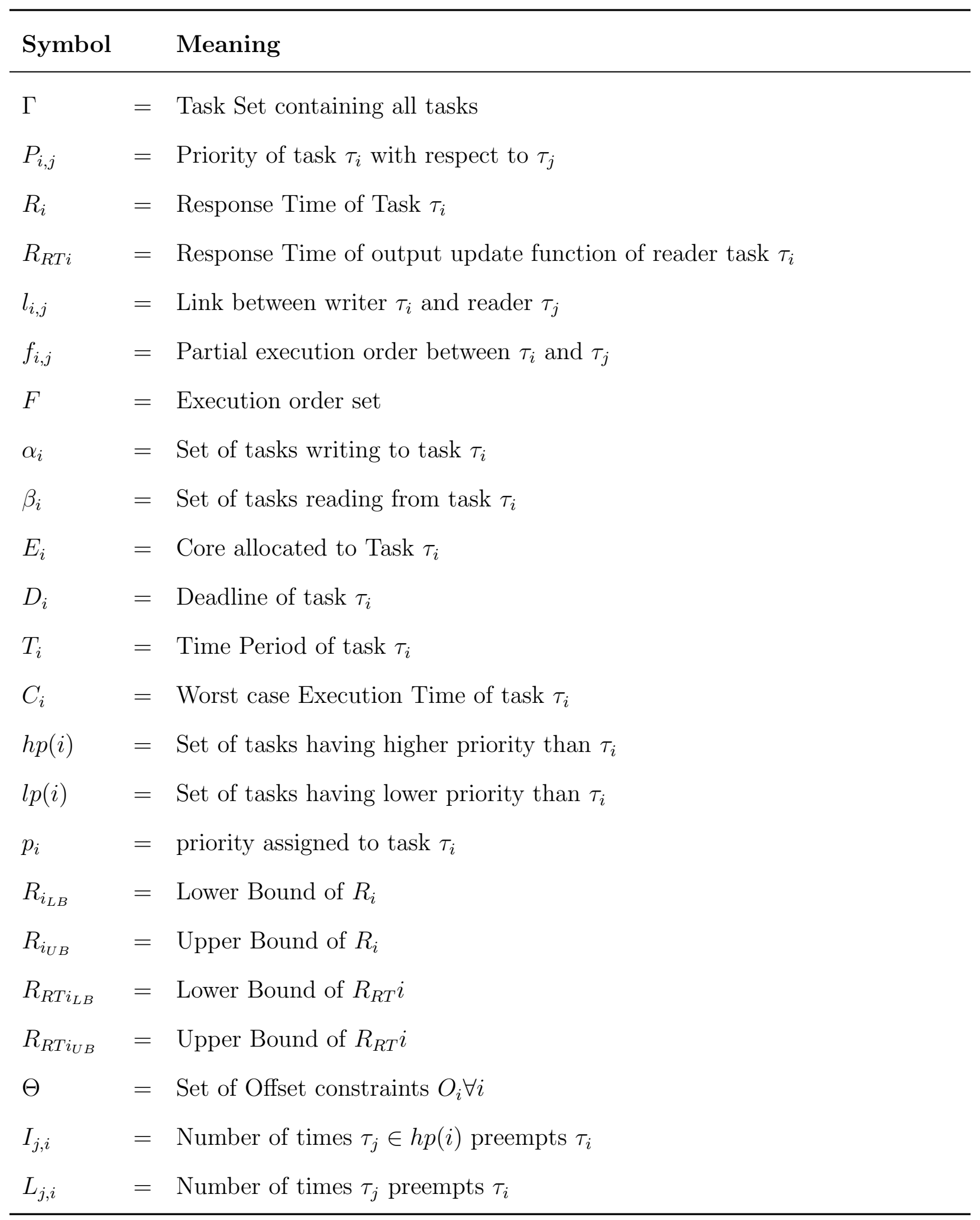

Table 5.1: Notations Used in our implementation 


\section{Constraint 1 - Valid Priority Order}

A binary decision variable $P_{i, j}$ is set to 1 , if and only if priority of task $\tau_{i}$ is greater than that of task $\tau_{j}$ while task $\tau_{i}$ and $\tau_{j}$ are on the same core i.e. $\tau_{i} \in h p(j)$. If $P_{i, j}=1$ then accordingly $P_{j, i}$ must be zero. This logical constraint can be given as:

$$
\forall \tau_{i} \neq \tau_{j} \wedge E_{i}=E_{j}: P_{i, j}+P_{j, i}=1
$$

From the definition of transitivity, if task $\tau_{i}$ has higher priority than task $\tau_{j}$ (i.e. $P_{i, j}=1$ ), and task $\tau_{j}$ has higher priority than task $\tau_{k}$ (i.e. $P_{j, k}=1$ ), then task $\tau_{i}$ has higher priority than $\tau_{k}$, i.e. $P_{i, k}=1$. This logical constraint can be linearized as:

$$
\forall \tau_{i} \neq \tau_{j} \neq \tau_{k} \wedge E_{i}=E_{j}=E_{k}: P_{i, j}+P_{j, k} \leq 1+P_{i, k}
$$

We add constraints (5.1) and (5.2) to ensure that the MILP finds a fixed solution to the priority assignment of the system $\Gamma$ where every task $\tau_{i}$ has a unique priority $p_{i}$. Now we move towards response time analysis to ensure that constraint (3.9) is satisfied.

\section{Constraint 2 - Response Time Computation}

During its execution task $\tau_{i}$ will be preempted by high priority tasks running on the same core. A integer decision variable $I_{j, i}$ is used to represent the number of times task $\tau_{i}$ will be preempted by task $\tau_{j} \in h p(i)$ during its execution time $R_{i}$. The constraint can be shown as:

$$
\forall \tau_{i} \neq \tau_{j} \wedge E_{i}=E_{j}: I_{j, i} \geq \frac{R_{i}}{T_{j}}
$$

By including the priority order $\left(P_{j, i}\right)$ as a design variable, we define a positive integer $L_{j, i}$ as the number of times a task $\tau_{j}$ preempts task $\tau_{i}$ on the same core. The given constraint can 
be linearized by using big M notation as shown below :

$$
\forall \tau_{i} \neq \tau_{j} \wedge E_{i}=E_{j}: L_{j, i} \geq I_{j, i}-\left(1-P_{j, i}\right)\left\lceil\frac{D_{i}}{T_{j}}\right\rceil
$$

Remark 5.1. For the case of $P_{j, i}=1$ the constraint (5.4) reduces to constraint (5.3), whereas for $P_{j, i}=0$, it reduces to $L_{j, i} \geq \frac{R_{i}}{T_{j}}-\left\lceil\frac{D_{i}}{T_{j}}\right\rceil$. Since $R_{i} \leq D_{i}$ and $L_{j, i}$ is a positive integer, for $P_{j, i}=0$ we get $L_{j, i} \geq 0$. Thus we see that $P_{j, i}=0$ adds no additional constraint, hence ensuring that the low priority tasks do not affect the execution time of $\tau_{i}$.

After the calculation of interference from preemption we calculate the response time as done in Section 3.2. As denoted by equation (5.4) the decision variable $L_{j, i}$ represents the times task $\tau_{j}$ interferes with task $\tau_{i}$. Thus the net response time for task $\tau_{i}$ considering intra core interferences, can be linearized as:

$$
\forall \tau_{i} \neq \forall \tau_{j} \wedge E_{i}=E_{j}: R_{i}=C_{i}+\sum_{j} L_{j, i} \cdot C_{j}
$$

For computing the response time of the output update function $R_{R T i}$, we follow the similar procedure above. We assume the WCET of RT output update function to be nearly 0 $\left(10^{-5}\right.$ for our analysis) and priority equal to that of task $\tau_{i}$. Thus, we obtain the following constraint for calculating $R_{R T i}$ :

$$
\begin{array}{r}
\forall \tau_{i} \neq \forall \tau_{j} \wedge: E_{i}=E_{j}: \\
I_{j, i} \geq \frac{R_{R T i}}{T_{j}} \wedge L_{j, i} \geq I_{j, i}-\left(1-P_{j, i}\right)\left\lceil\frac{D_{i}}{T_{j}}\right\rceil \wedge R_{R T i}=C_{R T i}+\sum_{j} L_{j, i} \cdot C_{j}
\end{array}
$$

Additionally, we need to ensure that the output update function finishes its execution before 
the reader task $\tau_{i}$. Thus, we add the following constraint:

$$
\forall \tau_{i}: R_{R T i} \leq R_{i}-C_{i}
$$

\section{Constraint 3 - Ensuring System Schedulability}

The system can be made schedulable by adding the following constraint:

$$
\forall \tau_{i}: R_{i}+O_{i} \leq D_{i}
$$

\section{Constraint 4 - Execution order}

For any two communicating tasks $\tau_{i}$ and $\tau_{j}$, we need to have a unique defined partial execution order, thus we add the following anti-symmetric constraint to denote the presence of a single execution order

$$
\forall t_{i, j}: t_{i, j}+t_{j, i}=1
$$

For tasks communicating on the same core, since $t_{i, j}=1$ implies priority order $P_{i, j}=1$, thus we can add transitivity constraint (5.2):

$$
\forall \tau_{i} \neq \tau_{j} \neq \tau_{k} \text { on the same core: } t_{i, j}+t_{j, k} \leq 1+t_{i, k}
$$

The design constraints imposed by the partial execution order are as following:

\section{-For Intra-core communication link}

By principles 1 and 2, partial execution order $f_{i, j} \equiv\left(t_{i, j}=1\right)$ avoids the use of a delay block and enforces constraint (3.5). Otherwise for the partial execution order $f_{j, i} \equiv\left(t_{j, i}=1\right)$, we add a unit delay block and constraint (3.6). This can be formulated with the following linear 
constraints

$$
\begin{array}{r}
\forall l_{i, j}: E_{i}=E_{j}: \\
O_{j} \leq O_{i}+t_{i, j} \cdot D_{j} \wedge O_{i} \leq O_{j}+t_{j, i} \cdot D_{i} \wedge D B_{i, j}=t_{j, i}
\end{array}
$$

Thus, we can see that for $t_{i, j}=1$ constraint (3.6) i.e. $O_{j} \leq O_{i}+D_{j}$ becomes a trivial constraint that is already included in constraint (5.8). Similarly, for $t_{j, i}=1$ constraint (3.5) i.e. $O_{i} \leq O_{j}+D_{i}$ becomes a trivial constraint.

\section{- Inter-Core communication Link :}

By principles 3 and 4 , partial execution order $f_{i, j} \equiv\left(t_{i, j}=1\right)$ avoids using a delay block and enforces constraint (3.7). Otherwise for the partial execution order $f_{j, i} \equiv\left(t_{j, i}=1\right)$, we add a unit delay block and constraint (3.8). This can be formulated with the following linear constraints:

$$
\begin{gathered}
\forall l_{i, j}: E_{i} \neq E_{j}: \\
O_{i}+R_{i} \leq O_{j}+t_{j, i} \cdot D_{i} \wedge O_{j}+R_{R T j} \leq O_{i}+t_{i, j} \cdot D_{j} \wedge D B_{i, j}=t_{j, i}
\end{gathered}
$$

Following the similar logic as shown for intra-core links, $t_{i, j}=1$ makes design constraint (3.8) for unit delay block trivial and $t_{j, i}=1$ makes the constraint (3.7) for direct-feedthrough trivial.

\section{Objective:}

The objective function of this MILP model is to minimize the weighted sum of number of delay blocks added by the execution order while ensuring all the above constraints are satisfied.

$$
\min _{\forall l_{i, j}} \sum w_{i, j} \cdot t_{j, i}
$$




\section{Chapter 6}

\section{Customized Optimization Algorithm}

In chapter 5, we presented an MILP formulation to solve the problem but as we can see from our results (shown in section 7.1), it is not scalable to larger systems. The major drawback with the MILP formulation is the computation of feasible priority assignment and offset assignment for the system. As the number of tasks increases, the model becomes too huge with the increasing number of constraints, thus making the MILP intractable for large systems. Hence, we develop a problem specific framework for this problem as shown below. In our approach we seek to reduce the computational burden on MILP by removing the

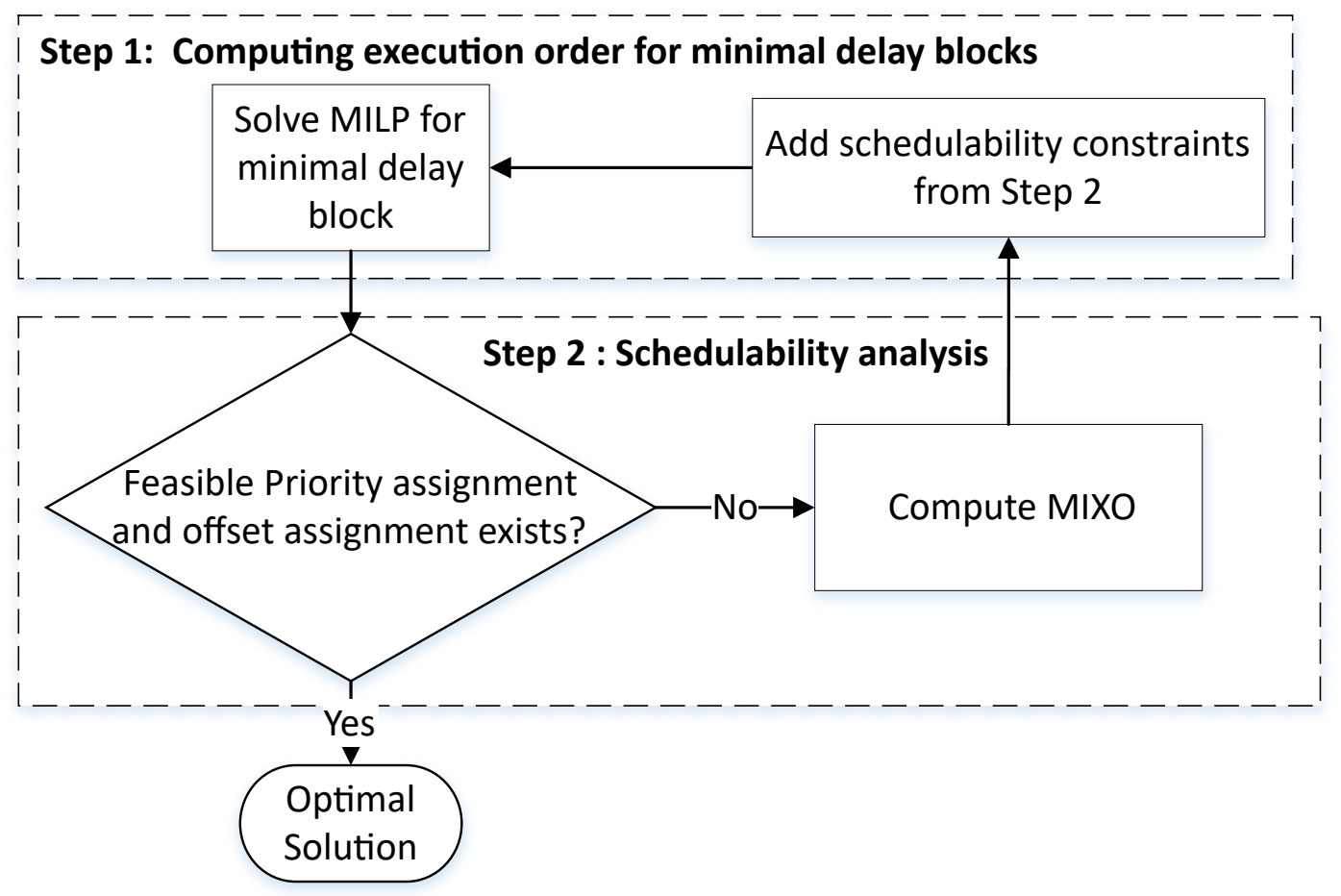

Figure 6.1: High Level Overview of Proposed Framework 
computation of response time assignment, offset assignment and priority assignment from the MILP. This computation is handled by a dedicated algorithm, which is more efficient than formulating a standard MILP. By doing so, we effectively divide the problem of obtaining the optimal execution order (as shown in equation (4.2)) into a two step iterative problem as shown in Figure 6.1. In step 1 we use a simplified MILP model that focuses on the objective and tries to impose directfeedthrough on as many links as possible, without considering any constraints for offset assignment or priority assignment. In step 2 we confirm that a feasible priority assignment and offset assignment exists that satisfies the design constraints enforced by the execution order obtained from step 1 (thus focusing on the schedulability part of equation (4.2)). Otherwise, we define the concept of Minimal Infeasible eXecution Order (MIXO) as an abstraction of the partial execution order that makes the system unschedulable. We use the results from step 2 to gradually shape the feasibility region of the MILP till we obtain an execution order which is schedulable. Thus essentially, we start with enforcing directfeedthrough on all links and during the runtime, we effectively learn which links require the addition of delay block for schedulability. The concept of using the schedulability constraints to shape feasibility of MILP is similar to that of the unschedulable core guided optimization presented by Zhao and Zeng [52]. In this section, we formally define MIXO and how we use it for our framework.

Definition 6.1. For a given system, we define execution order set $\boldsymbol{F}=\left\{f_{i_{1}, j_{1}}, . ., f_{i_{m}, j_{m}}\right\}$ as the set of the partial execution orders that exist across the communication links of the system. The number of elements in $F$ can be defined as its cardinality, denoted by $|\boldsymbol{F}|$.

Definition 6.2. To confirm that the system is schedulable for a given execution order $F$, we say that the task system $\Gamma$ is said to be $\boldsymbol{F}$-feasible if there exists

- a feasible priority assignment that satisfies the partial priority order $P_{i, j}=1$ for each element $f_{i, j}$ in $F$ by Principles 1 and 2 (as shown in equations (3.5) and (3.6)) 
- a feasible offset assignment that satisfies the offset constraints added by each element $f_{i, j}$ in $F$ by Principles 1, 2, 3 and 4 (as shown in equations (3.5), (3.6), (3.7) and (3.8)) along with the following constraint

$$
\forall i: R_{i}+O_{i} \leq D_{i}
$$

If the system is $F$-feasible, then we say that a feasible priority assignment and offset assignment exists that can satisfy all the design constraints imposed by $F$, thus ensuring schedulability. In this work, we use the terms like "feasible execution order", "F-feasibility" etc. to show that the system is $F$-feasible. Likewise, we say that the system "does not have feasible $F ", " F$-infeasibility" if the system is not $F$-feasible. The following example can elaborate on how we can determine if the system is $F$-feasible.

\begin{tabular}{|c|c|c|c|c|}
\hline$\tau_{i}$ & $T_{i}$ & $D_{i}$ & $C_{i}$ & $E_{i}$ \\
\hline 0 & 100 & 100 & 20 & 0 \\
1 & 100 & 100 & 40 & 0 \\
2 & 20 & 20 & 10 & 1 \\
3 & 200 & 200 & 96 & 1 \\
\hline
\end{tabular}

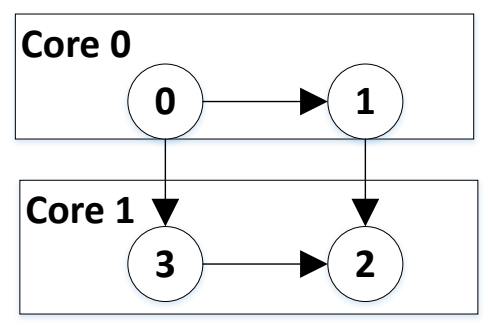

Table 6.1: Example Test Case

Example 6.3. Considering the Task System shown in Table 6.1, for execution order set $F=\left\{f_{0,1}, f_{2,1}, f_{3,2}, f_{3,0}\right\}$. The system $\Gamma$ is said to be $F$-feasible if it has a feasible priority assignment and an offset assignment that satisfies the constraints (3.5),(3.6),(3.7) and (3.8) 
added by each element in $F$ as shown.

$$
\begin{array}{r}
\text { For Intra Core Links : } \\
P_{0,1}=1 ; P_{3,2}=1 \\
O_{0} \leq O_{1} ; O_{3} \leq O_{2} \\
\text { For Inter Core Links : } \\
R_{R T 3}+O_{3} \leq O_{0} ; R_{R T 2}+O_{2} \leq O_{1} \\
\text { For Schedulability: } \\
\forall i: R_{i}+O_{i} \leq D_{i}
\end{array}
$$

In the given system, we see that $\Gamma$ is not $F$-feasible as priority order $P_{3,2}=1$ from $f_{3,2}$ does not return a feasible priority assignment. If task $\tau_{3}$ executes before $\tau_{2}$ i.e. if $f_{3,2}$ is enforced, then we get $R_{2}$ (from equation (3.3)) as 106. Thus we see that we say that the system is not $F$-feasible as $\tau_{2}$ misses its deadline $\left(R_{2}+O_{2}>D_{2}\right)$

Theorem 6.4. Let $F$ and $F^{\prime}$ be two execution order sets such that $F^{\prime} \subseteq F$, we can say that

$$
\Gamma \text { is } F \text {-feasible } \Rightarrow \Gamma \text { is } F^{\prime} \text {-feasible }
$$

Proof. Since, we can see from Principles 1, 2, 3 and 4 that each element in $F$ imposes constraints on a system $\Gamma$, a subset $F^{\prime}$ will impose fewer constraints on $\Gamma$ than $F$. Thus if a feasible priority assignment and offset assignment satisfies the constraints imposed by $F$, then it will also satisfy the constraints imposed by $F^{\prime}$.

Corollary 6.5. Thus by contrapositive law we have $F^{\prime} \subseteq F$

$$
\Gamma \text { is not } F^{\prime} \text {-feasible } \Rightarrow \Gamma \text { is not } F \text {-feasible }
$$


This essentially means that if the constraints imposed by $F^{\prime}$ make the system unschedulable, then the system will definitely be unschedulable for any superset of $F^{\prime}$. Now that we know how to confirm the schedulability of the system for a given execution order, we can reformulate the optimization problem as below.

\subsection{Problem Formulation for MIXO Framework}

From definition 6.2 , we can say that if the system is $F$-feasible, then a feasible priority assignment and offset assignment exists. From section 4, we see that for link $l_{i, j}$, partial execution order $f_{j, i} \equiv\left(t_{j, i}=1\right)$ denotes the presence of unit delay block and $f_{i, j} \equiv\left(t_{i, j}=1\right)$ denotes the absence of the delay block. In order to obtain the optimal execution order shown in equation (4.2), we formulate the problem as:

$$
\begin{array}{ll} 
& \min _{\forall F} \sum_{\forall l_{i, j}} w_{i, j} \cdot t_{j, i} \\
\text { s.t. } & \Gamma \text { is } F \text {-feasible } \\
& t_{i, j}+t_{j, i}=1, \forall i \neq j \\
& t_{i, j} \geq t_{i, k}+t_{k, j}-1, \forall i \neq j \neq k
\end{array}
$$

By reformulating the problem as above, we can split the problem into two parts such that MILP deals with the minimization of the objective and the feasibility analysis checks if the system is $F$-feasible (as mentioned in definition 6.2). From the perspective of the reformulated problem (6.5), Corollary 6.5 suggests that if a partial execution order set $F^{\prime}$ is known to be infeasible, then the search for the optimal feasible $F$ shall avoid any superset of $F^{\prime}$. Thus an infeasible partial execution order set $F^{\prime}$ provides a clue for performing search space reduction for the MILP. Intuitively, the smaller the $F^{\prime}$, the greater the number of infeasible partial execution order sets it can capture. To do so, we use the concept of MIXO as an 
irreducible abstraction of the execution order set that makes the system unschedulable. We define the concept of MIXO as shown below.

Definition 6.6. By using corollary 6.5 and definitions 6.1, 6.2, we define an execution order set $U$ as a minimal infeasible execution order set (MIXO) if and only if

- $\Gamma$ is not $U$-feasible

- $\forall F^{\prime} \subset U, \Gamma$ is $F^{\prime}$-feasible

Remark 6.7. By theorem 6.4, the second condition can be formulated as

$$
\forall F^{\prime} \subset U \text { s.t. }\left|F^{\prime}\right|=|U|-1, \Gamma \text { is } F^{\prime} \text {-feasible }
$$

Example 6.8. For system in Table 6.1, we see that for $F=\left\{f_{3,2}, f_{0,1}\right\}, \Gamma$ is not $F$-feasible as $P_{3,2}=1$ is not a feasible priority order. However $F$ is not a MIXO as $\Gamma$ is still infeasible for $F^{\prime}=\left\{f_{3,2}\right\}$, where $F^{\prime} \subset F$. But we see that $F^{\prime}$ is a MIXO as $\Gamma$ is not $F^{\prime}$-feasible but is $F^{\prime \prime}$-feasible where $F^{\prime \prime}=\{\phi\}$ is a subset of $F^{\prime}$.

For the computed MIXO $U$, by definition 6.6 all the enforced partial execution orders in $U$ cannot be simultaneously satisfied. Thus by using equation (6.6) we use the following constraint to obtain a subset of the enforced execution order set $F^{\prime}$ s.t. the system is $F^{\prime}$ feasible :

$$
\sum_{\forall f_{i, j} \in U} t_{i, j} \leq|U|-1
$$

By using the equation (6.7), we can add the feasibility constraints for each obtained MIXO to shape the feasibility region of the MILP. We see that for a given execution order set, we can obtain the MIXO as explained below. 


\subsection{MIXO Computation}

For a given execution order set and a system $\Gamma$, we use algorithm 1 to compute the minimal infeasible execution order set $F$. In algorithm 1 , we remove each partial execution order one by one to check if $\Gamma$ becomes $F$-feasible. If removing $f_{i, j}$ makes $\Gamma F$-feasible, we keep $f_{i, j}$ in $F$ as it is an infeasible execution order that makes the system unschedulable. However, if removing $f_{i, j}$ does not change the schedulability of the system, then we can say that it is not a cause of unschedulability. By iterating through all elements of $F$, the function returns a computed set of minimal infeasible execution order $F$ such that $\Gamma$ is not $F$-feasible but $\Gamma$ is $F^{\prime}$-feasible $\forall F^{\prime} \subset F$.

The following example shows how we can use Algorithm 1 to compute the MIXO for a given execution order set $F$.

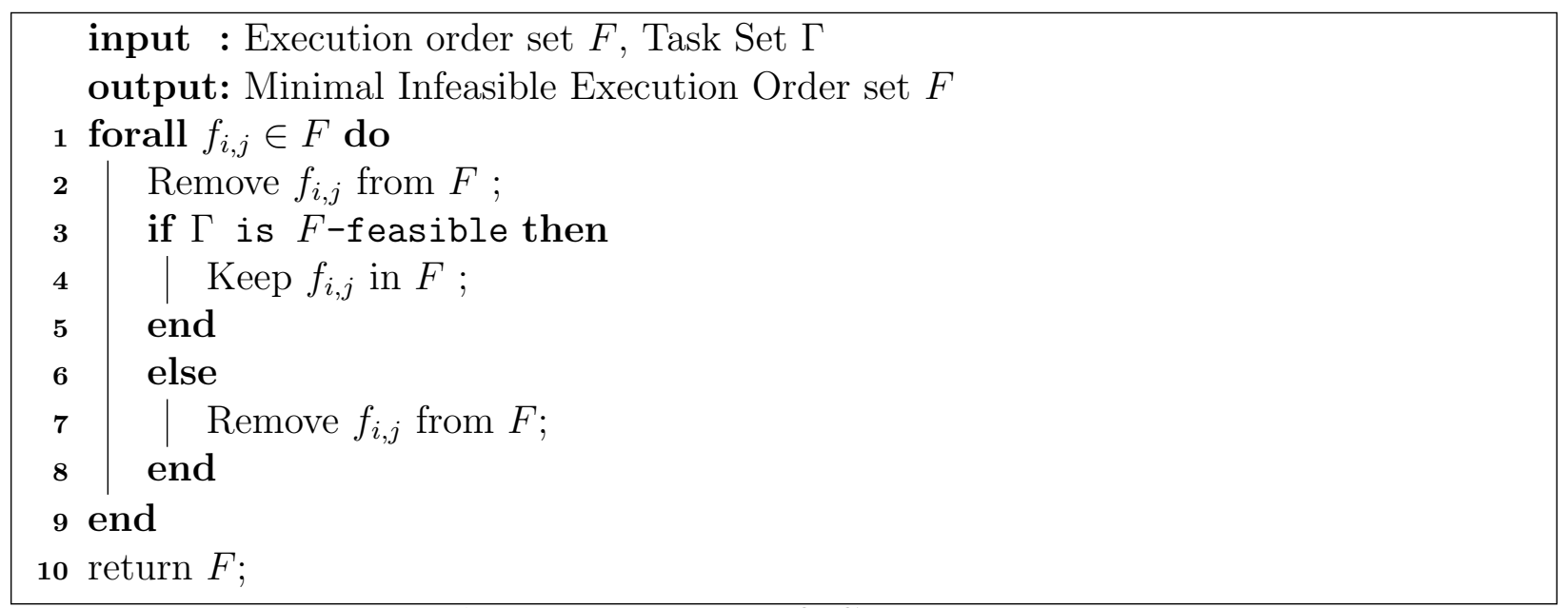

Algorithm 1: MIXO Computer

Example 6.9. Continuing from example 6.3, we see that $\Gamma$ is not $F$-feasible. By using algorithm 1, we start with $F=\left\{f_{0,1}, f_{2,1}, f_{3,2}, f_{3,0}\right\}$ and iteratively remove $f_{i, j}$ till we see that removing $f_{i, j}$ makes the system $F$-feasible.

We see that removing $f_{3,2}$ makes the system $F$-feasible s.t. $F=\left\{f_{3,0}\right\}$. Thus we put back $f_{3,2}$ in $F$ and continue to remove $f_{3,0}$. 
We see that removing $f_{3,0}$ does not make the system schedulable and hence remove $f_{3,0}$ from $F$. Thus, we get $f_{3,2}$ is obtained as the sole reason for unschedulability. We thus obtain the MIXO as $F=\left\{f_{3,2}\right\}$. Following equation 6.7 , the resultant schedulability constraint added to the MILP is $t_{3,2} \leq 0$.

From the algorithm 1, we can see that the primary step in computing MIXO is checking if the system is $F$-feasible. Thus for a given $F$, to compute a single MIXO we check the feasibility $|F|$ times. In the following section, we discuss how we can optimize this analysis to ensure faster computation of MIXO.

\subsection{Feasibility Analysis for MIXO Based Framework}

To check if the given system $\Gamma$ is $F$-feasible, we need to check if a feasible priority assignment and offset assignment exists as shown in definition 6.2. To do so, we need to formulate a mathematical formulation to ensure that all the constraints enforced by the execution order $F$ are satisfied. Thus for every element in $F$, we add the design constraints (3.5), (3.6) (3.7), (3.8) and (3.9) to confirm if a feasible priority assignment and offset assignment exists. For a given $F$, the offset assignment should satisfy the following constraints:

$$
\forall i: R_{i}+O_{i} \leq D_{i}
$$

For Intra core links from $\tau_{i}$ to $\tau_{j}$ :

$$
\begin{aligned}
& \forall f_{i, j}: O_{i} \leq O_{j} \\
& \forall f_{j, i}: O_{j} \leq O_{i}
\end{aligned}
$$

For Inter core links from $\tau_{i}$ to $\tau_{j}$ :

$$
\begin{array}{r}
\forall f_{i, j}: R_{i}+O_{i} \leq O_{j} \\
\forall f_{j, i}: R_{R T j}+O_{j} \leq O_{i}
\end{array}
$$


At the same time, the feasible priority assignment should satisfy the following constraint

For Intra core links from $\tau_{i}$ to $\tau_{j}$ :

$$
\begin{aligned}
& \forall f_{i, j}: P_{i, j}=1 \\
& \forall f_{j, i}: P_{j, i}=1
\end{aligned}
$$

\subsubsection{Exact Feasibility Analysis}

We call this feasibility analysis exact as it can find all the schedulability constraints required to obtain a feasible execution order set. For a system to be $F$-feasible, it needs a feasible offset assignment that satisfies the constraints shown in (6.8) as well as a feasible priority assignment that satisfies (6.9). Since solving this mathematical formulation can be quite complex, we use the concept of Maximal Unschedulable Deadline Assignment (MUDA) [51] to determine if a feasible priority assignment and offset assignment exists. MUDA based framework reformulates the problem into finding a schedulable virtual deadline assignment that satisfies the constraints in equation (6.8) and (6.9). Virtual deadline $\boldsymbol{d}_{\boldsymbol{i}}$ used in this framework is essentially the maximum feasible upper bound on the response time of a task and is defined as:

$$
\forall \tau_{i}: R_{i} \leq d_{i} \text { s.t. } C_{i} \leq R_{i} \leq D_{i}
$$

This framework essentially divides the problem into two components such that the MILP only solves for the constraints in (6.8) and the responsibility of assigning priorities satisfying (6.9) is left to another sub-function. The working of MUDA-framework is as following

1. A MILP is used to solve the constraints enforced for offset assignment shown in equation (6.8), denoted by $G(X) \leq 0$. This MILP returns a feasible virtual deadline assignment without worrying about priority assignment. However, if no such feasible deadline assignment exists then we return that the system is not $F$-feasible. 


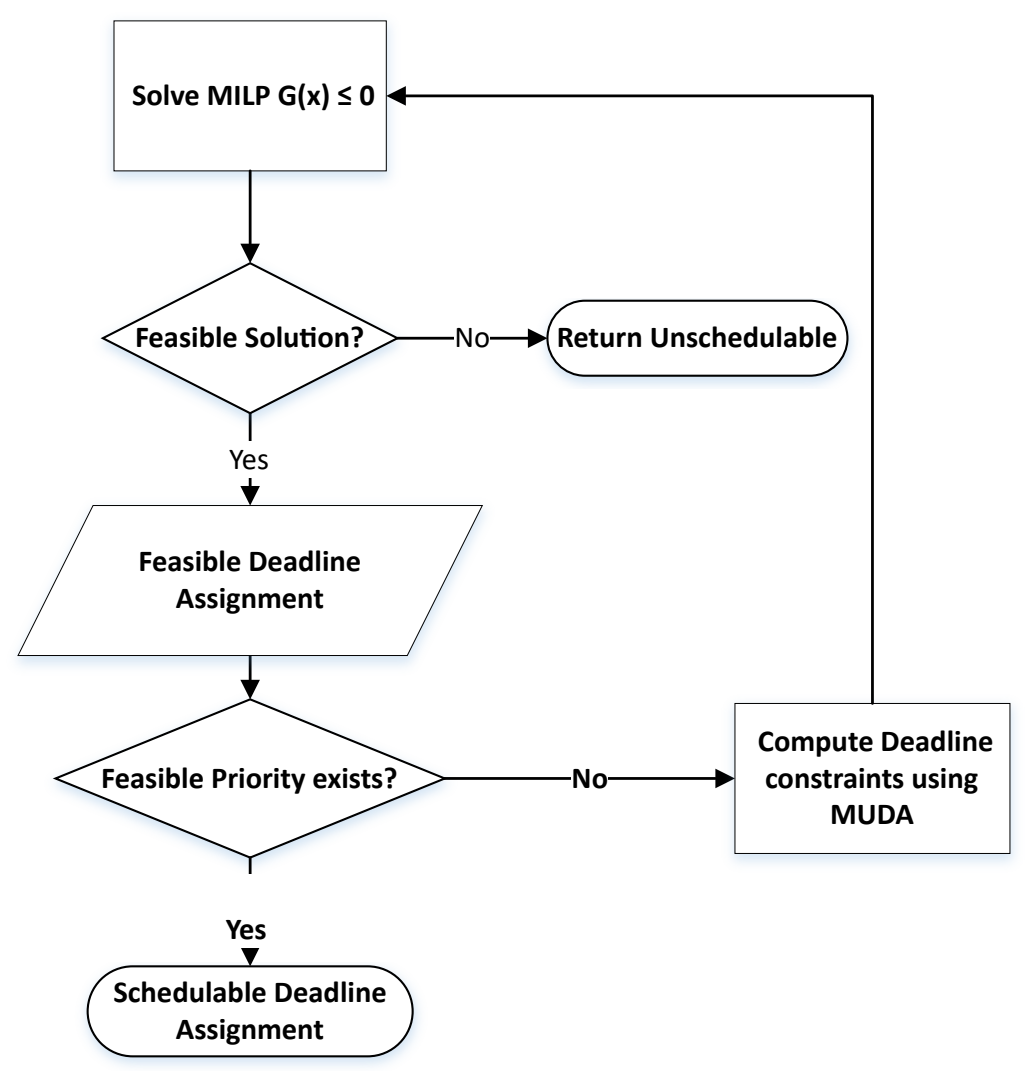

Figure 6.2: MUDA Framework Overview

2. We confirm if the system has a feasible priority assignment for the feasible deadline assignment obtained from MILP. Otherwise, we calculate MUDA as an abstraction of the virtual deadline assignment that makes the system unschedulable. This MUDA is then added as a deadline constraint to refine the MILP search space till a schedulable deadline assignment is obtained.

If the MUDA framework (as shown in Figure 6.2) can return a schedulable deadline assignment, we say that the system is $F$-feasible. To determine schedulability, we check if a feasible priority assignment exists, using revised version of Audsley's algorithm [4]. In our revised algorithm, we iteratively pick a task and try to assign it a priority $\boldsymbol{p}$, starting with the lowest priority first. While choosing the candidate, we need to ensure that (a) the response time of task at priority level $p$ (as calculated by equation (3.3)) is not greater than 
the virtual deadline computed by MILP and (b) the net priority assignment follows the partial order enforced by the execution order (as shown in equation (6.9)). For example, for $f_{i, j} \in F$, for intra-core links we need to ensure that we assign priority to task $\tau_{i}$ only if task $\tau_{j}$ has already been assigned a lower priority.

Example 6.10. For system shown in Table 6.1, with $F=\left\{f_{0,1}, f_{3,0} . f_{2,3}, f_{2,1}\right\}$, we use MUDAframework to check if the system is $F$-feasible. The MUDA framework reformulates the problem into two parts:

\begin{tabular}{c|c} 
Deadline constraints: & Partial priority order (PPO) constraints: \\
\hline$O_{1} \geq O_{0}$ & $p_{0}>p_{1}$ \\
$O_{2} \geq O_{3}$ & $p_{3}>p_{2}$ \\
$O_{2} \geq d_{1}+O_{1}$ & \\
$O_{3} \geq d_{0}+O_{0}$ & \\
$d_{i}+O_{i} \leq T_{i}, \forall \tau_{i}$ & \\
$D_{i} \geq d_{i} \geq C_{i} \wedge d_{i} \geq R_{i}, \forall \tau_{i}$ &
\end{tabular}

For the first iteration, the MILP solves the deadline constraints and provides the feasible virtual deadline assignment

$$
\left[d_{0}, d_{1}, d_{2}, d_{3}, d_{R T 2}, d_{R T 3}\right]=[20,40,10,96,0,10]
$$

Now, we use the revised Audsley's algorithm to check if a feasible priority assignment exists for deadline assignment (6.12). This priority assignment has to also satisfy the priority constraints in (6.11). Using our revised Audsley's algorithm, we determine that for feasible priority assignment, $\tau_{3}$ cannot have a virtual deadline less than 196 . Thus, we obtain MUDA 
as an abstraction of this unschedulable deadline and add it to the MILP as a constraint, to shape its feasibility region.

$$
d_{3} \geq 196
$$

In the next iteration the MILP considers constraint (6.13) and provides the virtual deadline assignment and the offset assignment as

$$
\begin{array}{r}
{\left[d_{0}, d_{1}, d_{2}, d_{3}, d_{R T 2}, d_{R T 3}\right]=[20,40,10,196,0,10]} \\
{\left[O_{0}, O_{1}, O_{2}, O_{3}\right]=[10,10,0,0]}
\end{array}
$$

Now, we see that the deadline assignment (6.14) satisfies all the constraints in (6.11) with a feasible priority assignment $p_{0}>p_{1}, p_{2}>p_{3}$ satisfying (6.11).

Since all the constraints enforced by $F$ provide a schedulable deadline assignment, we say that $\Gamma$ is $F$-feasible.

As mentioned in section 6.2 , the feasibility analysis is the primary bottleneck in our performance. While MUDA based approach is more efficient than formulating an MILP [51], it is still too expensive to be used for computing all MIXOs. Thus we decide to use a more relaxed feasibility analysis before using this analysis. By doing so, we compute a share of the MIXOs using a faster but more approximate analysis before using this slower but exact analysis.

\subsubsection{Necessary Feasibility Analysis 1}

In this form of feasibility analysis, we check the necessary conditions only, thus we opt for a reduced analysis for improved speed. Even though this analysis does not provide the complete set of MIXOs, it is much faster than the exact analysis presented in section 6.3.1. In this analysis we use algorithm 2 to determine if the system is schedulable for the the lowest 
possible offset assignment (denoted by $\Theta$ ) that can be computed from the execution order. We optimistically confirm that the system is $F$-feasible if a feasible priority assignment exists and if the system is schedulable with the lowest possible response time and offset assignment. To check the existence of a feasible priority assignment we use the revised version of Audsley's algorithm as discussed in the exact analysis mentioned in section 6.3.1. While determining the priority assignment, we assign priorities that ensure that (a) the response time of a task does not exceed the computed upper bound from algorithm 2 (i.e. $R_{i_{U B}}$ ) and (b) the partial priority order satisfies the constraints (6.9) imposed by the execution order.

Since our priority assignment is compliant with Audsley's algorithm, it satisfies the following three conditions [4] [51]

- The WCRT $R_{i}$ of any task $\tau_{i}$ calculated by our priority assignment does not depend on the relative order of tasks in $h p(i)$

- Similarly, the calculation of $R_{i}$ by our priority assignment does not depend on the relative order of tasks in $l p(i)$

- $R_{i}$ is monotonically increasing with the number of elements in $h p(i)$, i.e., if $\tau_{i}$ is dropped to a lower priority while the relative priority order of other tasks remains the same, $R_{i}$ will only increase.

By considering the above three conditions, we can compute the minimum response time of a task by assigning it the highest possible priority while ensuring that a feasible priority assignment exists. We use this computed minimum response time to ensure minimum offset carryover, thus allowing us to compute the minimum offset assignment $\Theta$ that satisfies the constraints in (6.8) (as done in Lines 18, 22).

From the computed minimum offset assignment $\Theta$ we adjust the upper bound of response time of all tasks $\left(R_{i_{U B}} \forall \tau_{i}\right)$ to minimize the range of feasible response time values (as shown 
in Line 31). Since the offset computed is the lowest possible, the response time cannot exceed this computed upper bound while satisfying the constraint (3.9) for schedulability.

We iterate through the algorithm 2 till we converge on a fixed offset assignment or till the system returns unschedulability (Lines 28 and 9). Since this analysis considers all the constraints enforced by execution order, it helps in computing a major share of the schedulability constraints before we use the exact analysis.

Example 6.11. For system $\Gamma$ in Table 6.1 , with $F=\left\{f_{0,3}\right\}$ we use algorithm 2 to check if the system is $F$-feasible. By Lines 1-5, we initialize the values as

$$
\begin{array}{r}
{\left[O_{0}, O_{1}, O_{2}, O_{3}\right]=[0,0,0,0]} \\
{\left[R_{0_{L B}}, R_{1_{L B}}, R_{2_{L B}}, R_{3_{L B}}\right]=[20,40,10,96]} \\
{\left[R_{0_{U B}}, R_{1_{U B}}, R_{2_{U B}}, R_{3_{U B}}\right]=[100,100,20,200]}
\end{array}
$$

The design constraint imposed in algorithm 2 by $F$ is as following

$$
O_{3}=\max O_{3}, O_{0}+R_{0_{L B}}
$$

A feasible priority assignment for the system exists as $p_{0}>p_{1}, p_{2}>p_{3}$ for Line 8 .

By Line 17, we compute lower bound response time values with no partial priority order constraints as

$$
\left[R_{0_{L B}}, R_{3_{L B}}\right]=[20,196]
$$

By Line 18, we compute the lowest possible offset values as mentioned by the design constraint (6.16)

$$
O_{3}=20 ; O_{0}=0
$$

Thus, we see that the algorithm 2 returns unschedulability at Line 27 as $R_{3_{L B}}+O_{3}>D_{3}$. 


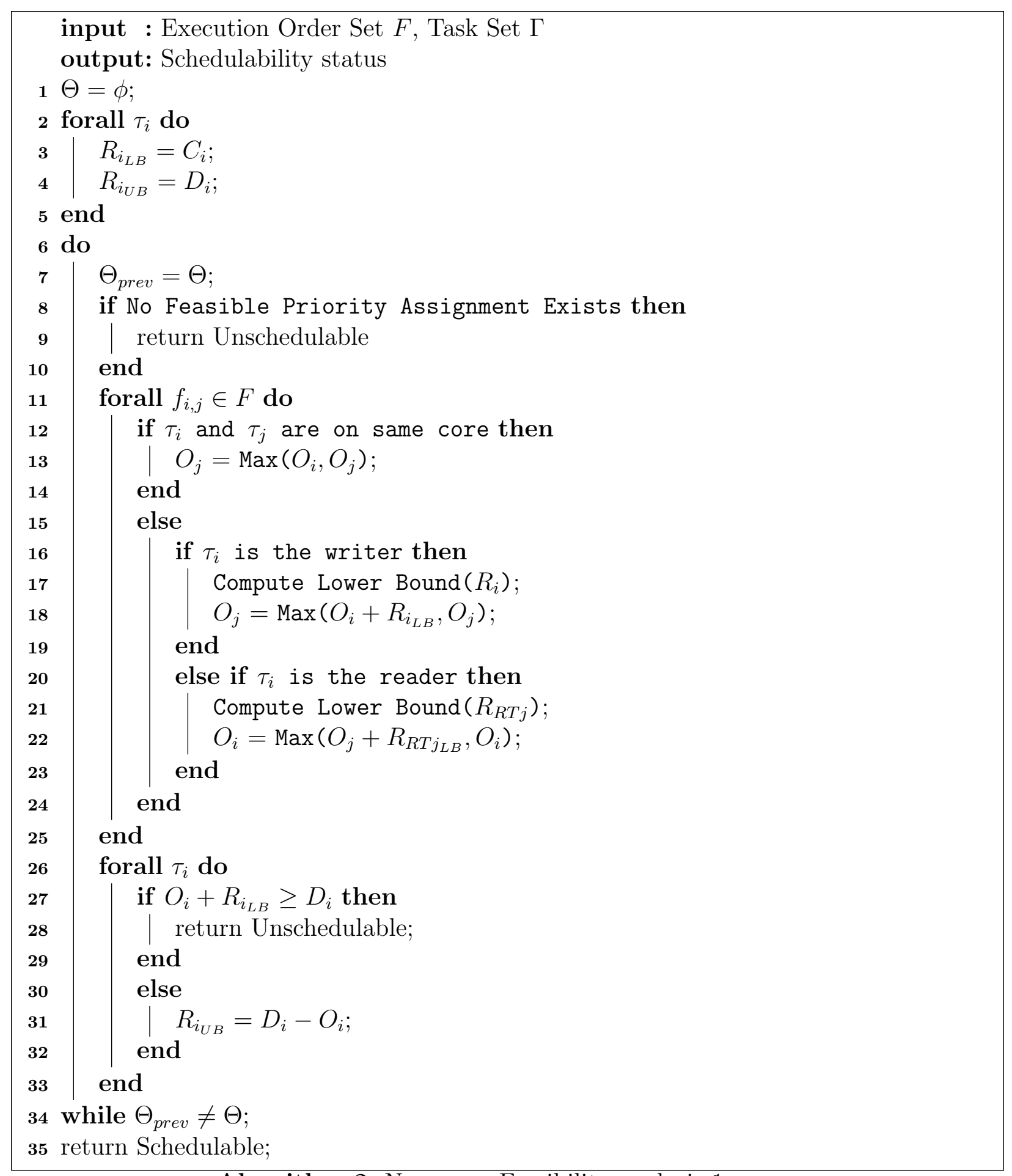

Algorithm 2: Necessary Feasibility analysis 1 
Thus we say that the system is not $F$-feasible.

However, $F_{1}=\left\{f_{3,0}\right\}$ makes $\Gamma F_{1}$-feasible. The design constraint imposed is

$$
O_{0}=\max O_{0}, O_{3}+R_{R T 3_{L B}}
$$

By Lines 17 and 21, we compute lower bound response time values with no partial priority order constraints as

$$
\left[R_{0_{L B}} R_{R T 3_{L B}}\right]=[20,10]
$$

By Line 18 and equation (6.19), we compute the lowest bound offset values $\left[O_{0}, O_{3}\right]=[10,0]$ We see that $R_{0_{L B}}+O_{0} \leq D_{0}$ with a feasible priority assignment $p_{0}>p_{1}, p_{2}>p_{3}$. Upon reiterating, we see that the computed offset values do not change. Thus, we say that the system is $F_{1}$-feasible as there exists a feasible priority assignment and the system is schedulable for the lowest possible response time and offset assignment.

Even though this analysis cannot compute all MIXOs as it optimistically checks for feasibility, it helps in computing obviously infeasible execution orders before exact analysis can be used. Furthermore, we observe that the basic feasibility analysis for both exact analysis and necessary analysis 1 , involves checking if a feasible priority assignment exists. Thus for an even more primitive analysis we can check if a feasible priority assignment exists before using the above mentioned necessary analysis 1 or the exact analysis.

\subsubsection{Necessary Feasibility Analysis 2}

In this feasibility analysis, we further simplify the feasibility condition to simply determine if a feasible priority assignment exists. In this feasibility test we do not consider offset assignment but rather just check if the partial priority order enforced by $F$ can return a feasible priority assignment. To determine if such a priority assignment exists we simply use 
a revised version of Audsley's algorithm as mentioned in exact feasibility analysis (mentioned in section 6.3.1). We compute this assignment, by iteratively assigning priorities to tasks starting with lowest priority first, such that (a) the response time of tasks does not exceed their deadlines and (b) the partial priority order satisfies constraints (6.9) imposed by the execution order. Since this analysis considers only restrictions for intra-core links, it is quite fast as compared to necessary analysis 1 and the exact analysis. However, this also means that it is the most flexible test and hence will fail to find as many MIXOs as necessary analysis 1 or exact analysis.

\subsubsection{Final Algorithm for Feasibility Analysis}

For developing an efficient dedicated algorithm for feasibility analysis we use all the aforementioned schedulability tests in a cascaded form as shown in Figure 6.3 (Steps 3-5). We start with necessary analysis 2 to check if a feasible priority assignment exists. If $\Gamma$ is not $F$-feasible at this level, we say that a feasible priority assignment does not exist for the given execution order set, and hence we compute MIXO accordingly. If $F$ passes the feasibility test using necessary analysis 2 , we use necessary analysis 1 for a stricter feasibility test. Finally if the system is $F$-feasible for both necessary analysis, we use exact analysis for a complete feasibility test. By using this multi-layered approach, we see that the necessary analysis 1 and 2 shape the majority of the schedulability region of the MILP and thus significantly

reduce the computation load on exact analysis. Since using exact analysis itself is more efficient than MILP formulation [51], using this multi-layered approach guarantees to provide a much better run-time as compared to using MILP formulation. 


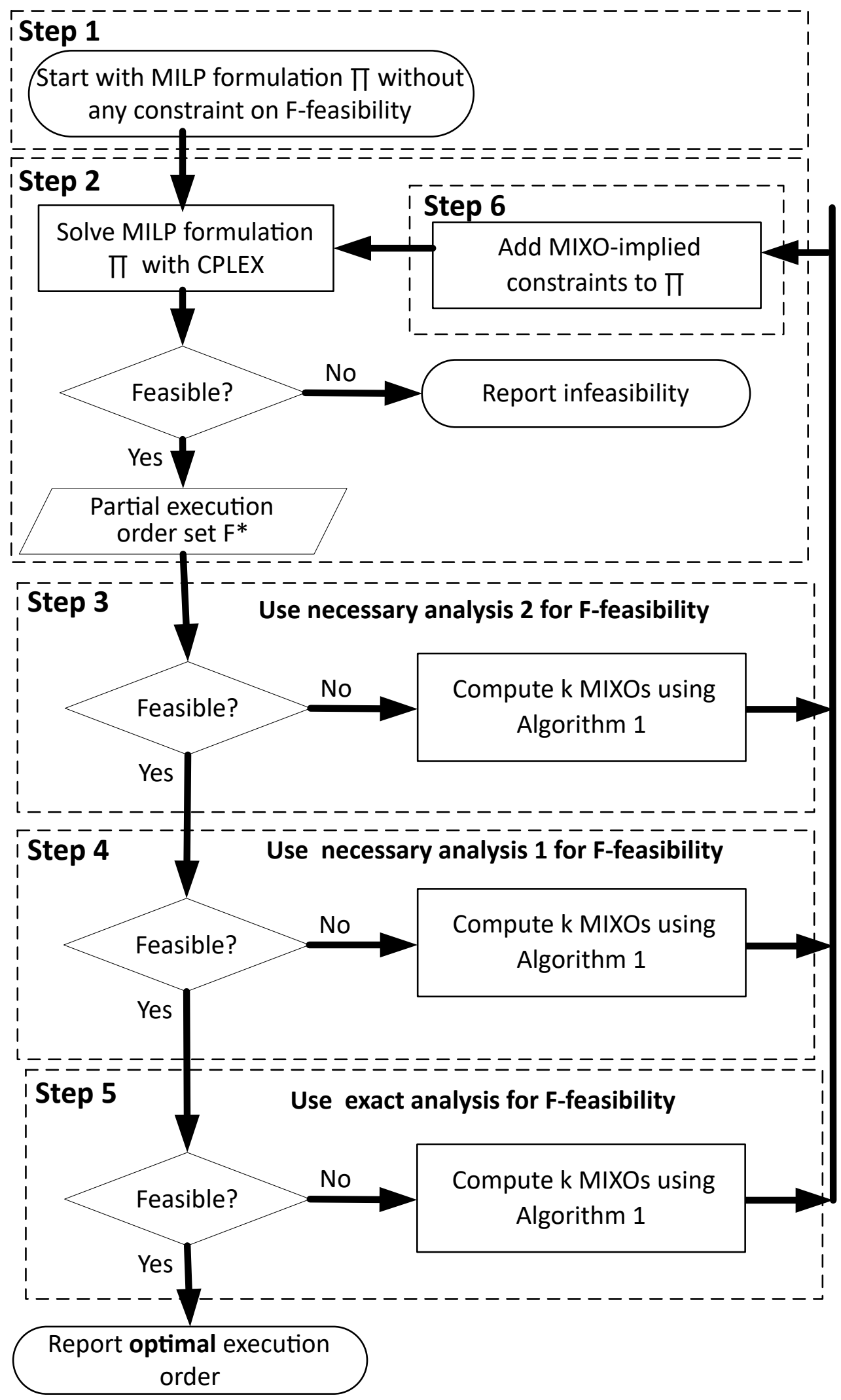

Figure 6.3: MIXO-guided Framework 


\subsection{MILP for MIXO Based Framework}

Following the reformulated problem as shown in equation (6.5), the simplified MILP $\Pi$ in our framework focuses on finding optimal execution order as shown below

$$
\begin{array}{r}
\min \sum_{\forall l_{i, j}} w_{i, j} \cdot t_{j, i} \\
\forall l_{i, j}: t_{i, j}+t_{j, i}=1 \\
\forall \tau_{i} \neq \tau_{j} \neq \tau_{k} \text { on the same core: } t_{i, j}+t_{j, k} \leq 1+t_{i, k}
\end{array}
$$

To ensure that the obtained execution order is valid, we add the anti-symmetric constraint (5.9) and the transitivity constraint (5.10) on the partial execution order. By removing the abstraction of offset and priority assignment from the MILP, we simplify the MILP model considerably. Furthermore, we see that the MILP in (6.21) only has binary variables, which further reduces the computational complexity. To ensure that the execution order is feasible, this MILP uses MIXO-implied constraints (as shown in (6.6)) to define its search space. In the following section, we see how this MILP works with the dedicated algorithm for feasibility analysis to obtain the optimal execution order (as shown in Figure 6.3).

\subsection{Putting All Together - MIXO-Guided Framework}

In order to solve the optimization problem discussed in section 4, we reformulate the original problem (equation (4.2)) into a MIXO-guided form (equation (6.5)) as shown in section 6.1. In MIXO-guided framework as shown in Figure 6.3, we reduce the computation in MILP by abstracting the feasibility analysis out of MILP (defined in section 6.4) into a separate function (defined in section 6.3.4). Thus the MILP focuses on providing an execution order that adds minimal delay in the system and the feasibility analysis iteratively shapes the MILP's 
feasibility region using MIXO-implied constraints. One issue of designing a framework based on MIXO-implied constraint (6.6) for modeling the feasibility regions is that the total number of MIXOs grows exponentially w.r.t. the size of the system. For example, for a system with $m$ communication links, which suggests $2 m$ partial execution orders necessary to consider, the total number of MIXOs can be $O\left(2^{2 m}\right)$. Modeling the entire feasibility region would need to enumerate all MIXOs and their implied constraints, which is obviously impractical for large systems. However, this is rarely necessary. We observe that in most cases, the optimization objective is only sensitive to a small number of MIXOs. Thus, we propose an iterative refinement procedure that selectively explores and adds MIXO-implied constraints guided by the optimization objective, i.e., to derive and enforce MIXO-implied constraints only when the optimization algorithm returns infeasible solutions (i.e., the returned partial execution order set is infeasible). The framework works by iterating through the below steps till the MILP provides an execution order that passes all the levels of feasibility analysis.

Step 1: Start with no schedulability constraints: We define the problem as done in section 6.1 with no initial constraints for feasibility of the execution order.

Step 2: Solving the MILP П: To do so, we use the MILP defined in section 6.4. This MILP returns an execution order set $F^{*}$ which adds minimal delay in the system such that $F^{*}=\left\{f_{i, j} \mid t_{i, j}=1\right\}$. However, if no such execution order exists i.e. if the MILP cannot find a feasible solution, we return that the system is infeasible. This might happen during the run-time, if the utilization is too high to obtain any feasible priority assignment.

Step 3: Use Necessary Analysis 2: In this step we confirm if a feasible priority assignment exists for the computed execution order. Otherwise, we compute k MIXOs that are added as constraints back to the MILP. To limit the run-time of each iteration, we allow a maximum of 5 schedulability constraints to be computed for a given execution order set. If the execution order is feasible for necessary analysis 2, we move to Step 4

Step 4: Using Necessary Analysis 1: Similar to Step 3, we use necessary analysis to check if 
the execution order is feasible. If it is feasible we continue to Step 5, otherwise we compute the corresponding MIXO-implied constraints to be added to the MILP.

Step 5: Using Exact Analysis: Since the execution order is feasible for both necessary analysis 1 and 2, we do a complete feasibility test using exact feasibility analysis. In case of infeasibility, MIXOs are computed and added as constraints to MILP. Otherwise, we return that the obtained execution order is optimal.

Step 6: Adding MIXO-implied constraints: In this step, we add MIXO-implied constraints as shown in equation (6.6) to shape the feasibility region of the MILP.

Thus by iterating through the above mentioned steps, we compute the optimal execution order for a given system. Furthermore, as seen from section 4, once we obtain the optimal execution order we can iterate through its elements and find the corresponding minimal delay block assignment.

Remark 6.12. In Step 2, it is possible that problem $\Pi$ becomes infeasible at some point. This happens for example, when the utilization of the system is so high that no priority assignment can schedule it. In this case, given any partial execution order set $F$, Algorithm 1 always returns $F=\emptyset$. The MIXO-implied by an empty set would be $0 \leq-1$, which causes $\Pi$ to be infeasible.

The algorithm is also guaranteed to terminate. This is because the number of MIXOs is finite, and during each iteration between Steps 2-6, the algorithm will find new MIXOs that are different from known ones. Finally, if a solution is deemed feasible at Step 5, it must be optimal with respect to the original problem, as it is optimal to a relaxed problem $\Pi$ : $\Pi$ only includes the implied constraints of a subset of all MIXOs.

We can see the working of this framework from the example below.

Example 6.13. We consider the system $\Gamma$ shown in Fig 6.1 with the assumption that the cost of delay block addition on all links is 1 i.e. $\forall l_{i, j}: w_{i, j}=1$. We now see, how our 
MIXO-guided framework solves the problem of optimal execution order for $\Gamma$.

Iteration 1 : Step 1 :We first initialize the problem into the problem of finding an optimal execution order as shown below:

$$
\begin{array}{r}
\min t_{1,0}+t_{3,0}+t_{2,1}+t_{2,3} \\
\text { s.t. } \Gamma \text { is } F \text {-feasible }
\end{array}
$$

where $F=\left\{f_{i, j} \mid t_{i, j}=1\right\}$

Step 2 : We formulate the MILP $\Pi$ as shown in (6.23)

$$
\begin{array}{r}
\min t_{1,0}+t_{3,0}+t_{2,1}+t_{2,3} \\
\forall l_{i, j}: t_{i, j}+t_{j, i}=1
\end{array}
$$

Solving $\Pi$ returns the execution order $F^{*}=\left\{f_{0,1}, f_{0,3}, f_{1,2}, f_{3,2}\right\}$

Step 3 : The system $\Gamma$ is not $F^{*}$-feasible for necessary analysis 2 , as a feasible priority assignment is not possible with partial priority order $P_{3,2}=1$. Thus we compute the MIXO $U_{1}=\left\{f_{3,2}\right\}$ using algorithm 1

Step 6: We use the computed $U$ to add the schedulability constraint as shown below.

$$
t_{3,2} \leq 0
$$

Iteration 2: Step 2: We solve the MILP (6.23) with constraint (6.24) and thus get the following solution $F^{*}=\left\{f_{0,1}, f_{0,3}, f_{1,2}, f_{2,3}\right\}$

Step 3: We see that $\Gamma$ is $F^{*}$-feasible for necessary analysis 2 as a feasible priority assignment $p_{0}>p_{1}, p_{2}>p_{3}$ that satisfies the design constraints imposed by $F^{*}$.

Step 4: We see that the system is not $F^{*}$-feasible by using necessary analysis 1 (as shown in example 6.11). The computed MIXOs are $U_{2}=\left\{f_{0,3}\right\}$ and $U_{3}=\left\{f_{1,2}\right\}$ 
Step 6: The schedulability constraints thus computed from $U_{2}$ and $U_{3}$ are

$$
\begin{aligned}
& t_{1,2} \leq 0 \\
& t_{0,3} \leq 0
\end{aligned}
$$

Iteration 3: Step 2: We solve MILP (6.23) with constraints (6.24) and (6.25) thus giving us the solution $F^{*}=\left\{f_{0,1}, f_{3,0}, f_{2,1}, f_{2,3}\right\}$.

Step 3: We see that $\Gamma$ is $F^{*}$-feasible for necessary analysis 2

Step 4: We see that $\Gamma$ is $F^{*}$-feasible for necessary analysis 1 as the system is schedulable with the computed lowest possible offset assignment and response time.

Step 5: We see that $\Gamma$ is $F^{*}$-feasible (as shown in example 6.10). Thus we say that $F^{*}$ is the optimal execution order for our system $\Gamma$.

We can now determine the delay block assignment from the obtained $F$ by using principles 1, 2, 3 and 4. Thus iterating for each element in $F$, we get the delay block assignment as $D B_{0,1}=0, D B_{1,2}=1, D B_{0,3}=1, D B_{3,2}=1$. 


\section{Chapter 7}

\section{Results}

In this section, we present the results of our experimental evaluation. We compare between the proposed MIXO-guided optimization framework and the MILP solution. Our experiments consists of two parts. The first evaluates on randomly generated systems with different settings and the second evaluates on an industrial case study of fuel injection system. The two techniques are compared in terms of run-time and the number of unit delay blocks added.

\subsection{Experiment on Random Systems}

This experiment aims to evaluate the performance of MIXO-guided optimization and MILP for systems across a wide range of settings. The first part of the experiment evaluates w.r.t systems of different number of tasks. We use Task Graphs For Free (TGFF) [19] for generating random acyclic directed graphs. The maximum number of writers to each task is limited to 3 and the maximum number of readers are 2. We consider dual-core platform (2 processors). System total utilization is randomly selected from interval $[1.4,1.8]$. Number of tasks varies from 10 to 70, which are then randomly and evenly distributed to the two processors. Utilization of each task is generated using UUnifast-Discard algorithm [14]. Periods are randomly chosen from a predefined set of values $\{5,10,20,40,50,100,200,400,500,1000\}$, which contains all the periods for the real-world automotive benchmark in [29]. To avoid excessive waiting time on difficult systems, we set a time limit of 30min for both techniques. 


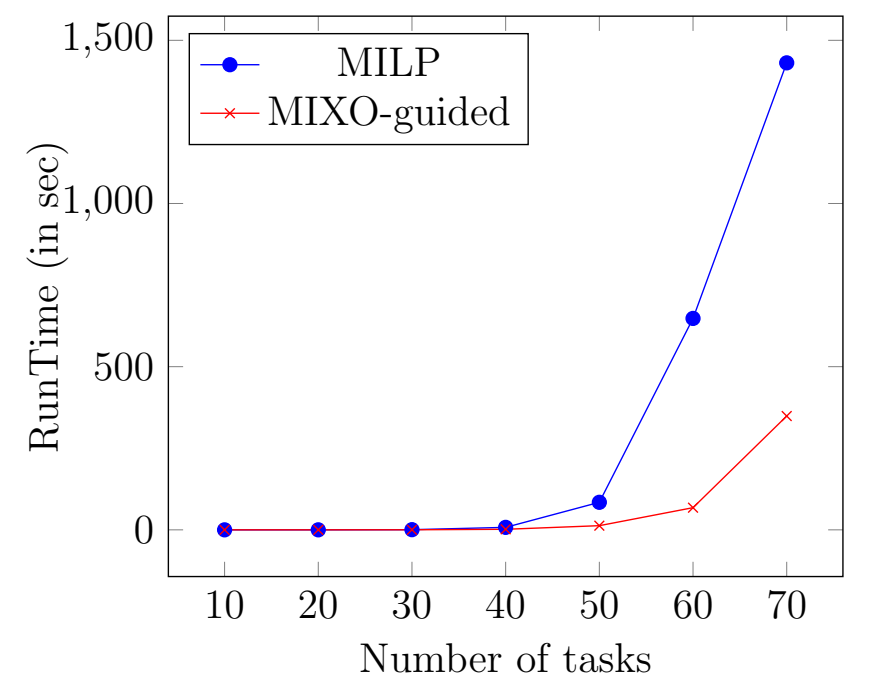

Figure 7.1: Average Run-Time for 1000 Random Systems

The average run-time by the two techniques is summarized in Figure 7.1. Each data point in the figure represents the average out of 1000 randomly generated systems. For systems with up to 50 tasks, MIXO-guided optimization and MILP have comparable performance. However, for larger systems comprising of 60 and more tasks, the complexity of MILP drastically increases. MIXO-guided optimization technique proves to 10X faster than MILP for 60-task systems. Furthermore, for 70-task systems, MILP timeouts for $65 \%$ of cases while MIXO-guided optimization technique only timeouts for $6 \%$. Thus, we can see that the proposed MIXO-guided framework is more scalable than MILP.

We then fix the total number of tasks to be 50, and check how the runtime of the algorithms vary with respect to a given system utilization. Hence, we fix the system utilization to be 1.2 to 1.8 , and collect the average runtime of both algorithms. Figure 7.2 illustrates the results. As in the figure, MIXO-guided framework always runs faster than MILP, and the gap in their algorithm efficiency becomes larger for higher utilization: at $180 \%$ system utilization (averagely 90\% on each core), MIXO-guided is about 15 times faster than MILP. As mentioned in section 6.3, the primary reason that MIXO-guided framework performs 


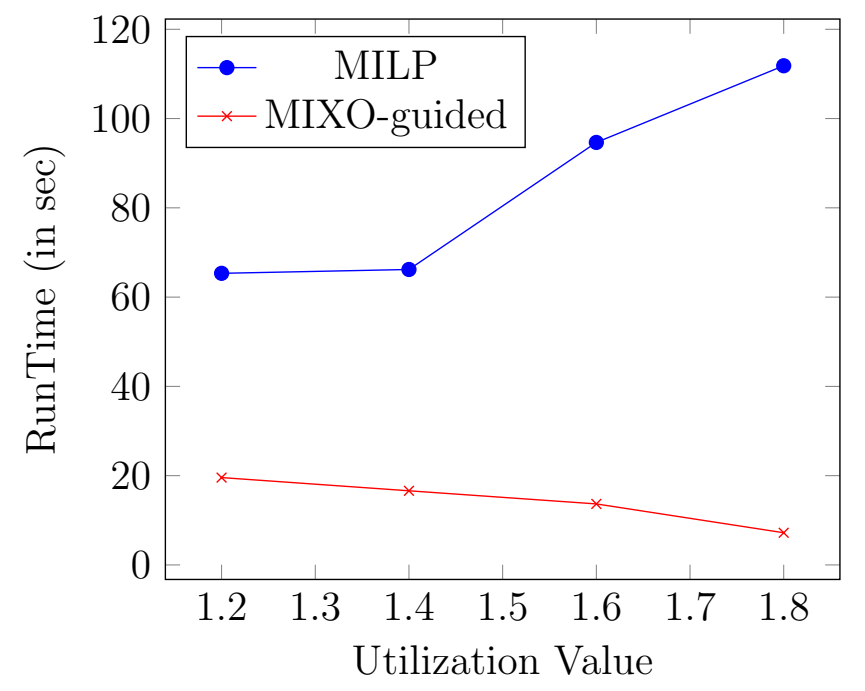

Figure 7.2: Average Run-Time for 50 tasks vs Utilization

\begin{tabular}{|c|c|c|}
\hline Analysis & \% of MIXOs computed & Time to compute single MIXO \\
\hline Exact Analysis & $22 \%$ & $1.145 \mathrm{sec}$ \\
\hline Necessary Analysis 1 & $37 \%$ & $0.014 \mathrm{sec}$ \\
\hline Necessary Analysis 2 & $41 \%$ & $0.08 \mathrm{sec}$ \\
\hline
\end{tabular}

Table 7.1: Comparing performance of each Analysis

better is that we shift the feasibility analysis into a much more efficient algorithm. We can see that our dedicated algorithm for feasibility analysis performs much better than using MILP formulation from the results obtained in Table 7.1 for 50 tasks. As mentioned in [51], the exact analysis itself is much faster than the MILP formulation. Furthermore, the results from Table 7.1 shows that the necessary analysis 1 and necessary analysis 2 perform upto 2 orders of magnitude faster than the exact analysis. At the same time, cumulatively they shape $78 \%$ of the feasibility region with their computed MIXOs, before shifting to the exact analysis. Thus, we can see that using the faster approximate analysis to compute the majority of MIXOs before the expensive exact analysis (as shown in Figure 6.3) greatly improves our run-time performance compared to MILP. 


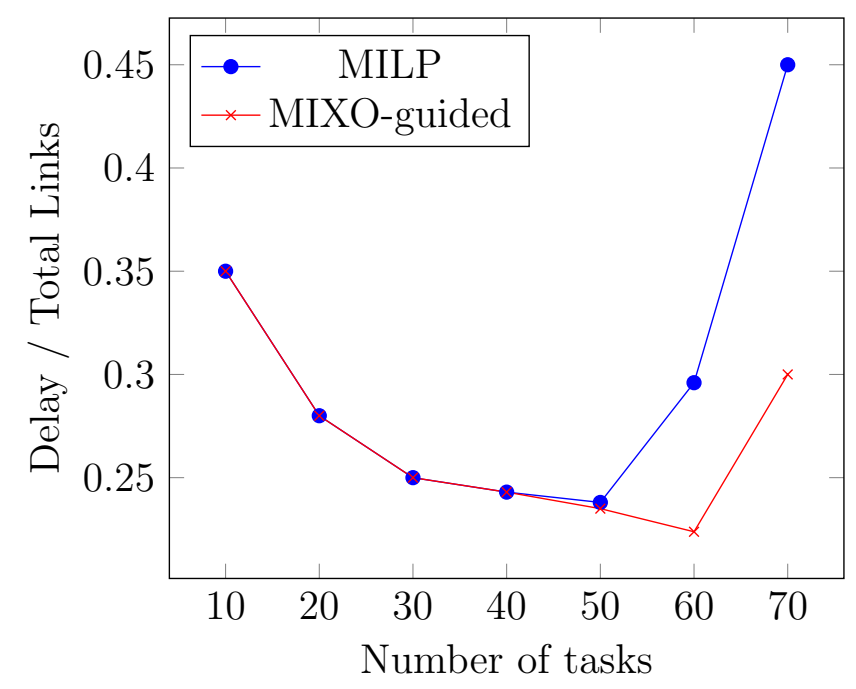

Figure 7.3: Normalized Objective Analysis for 1000 Random systems

Next we compare the average normalized objective by the two techniques. The normalized objective is defined as the objective value (the minimum number of unit delay RT blocks) over the total number of communication links. Two special cases need to be considered. If a technique fails to find any solution within the time limit, we consider the normalized objective to be 1 as a penalty. If a technique is capable of detecting infeasibility within the time limit, we consider the normalized objective to be 0 . Intuitively, the average normalized objective reflects the optimization quality of an algorithm. The results are summarized in Figure 7.3. The two techniques perform comparably for small systems of no larger than 50 tasks. For larger system of 60 and more tasks, MIXO-guided technique gives much better optimization result than MILP. This is mainly due to a large number of cases where MILP either timeouts without finding any feasible solutions or finding only sub-optimal solutions. The results of these experiments on random systems can be seen in a tabular form in Table 7.2. 


\begin{tabular}{|c|c|c|c|c|c|c|}
\hline \multirow{2}{*}{$\begin{array}{l}\text { Task } \\
\text { Count }\end{array}$} & \multicolumn{2}{|c|}{ Average Run-Time (in sec) } & \multicolumn{2}{c|}{ Average Normalized Objective } & \multicolumn{2}{c|}{ Total Timeouts } \\
\cline { 2 - 7 } & MILP & MIXO-guided & MILP & MIXO-guided & MILP & MIXO-guided \\
\hline 10 & 0.003 & 0.001 & 0.35 & 0.35 & 0 & 0 \\
\hline 20 & 0.047 & 0.023 & 0.28 & 0.28 & 0 & 0 \\
\hline 30 & 0.51 & 0.2 & 0.25 & 0.25 & 0 & 0 \\
\hline 40 & 7.62 & 1.72 & 0.243 & 0.243 & 1 & 0 \\
\hline 50 & 84.42 & 12.811 & 0.238 & 0.235 & 10 & 0 \\
\hline 60 & 647.86 & 67.945 & 0.296 & 0.223 & 180 & 1 \\
\hline 70 & 1430.97 & 348.7 & 0.45 & 0.30 & 651 & 62 \\
\hline
\end{tabular}

Table 7.2: Results for 1000 Random Systems

\subsection{Fuel Injection System Case Study}

Our second experiment evaluates on an industrial case study of a simplified fuel-injection system [18]. The system contains 90 tasks with 106 communication links. The case study was originally configured to run on a single core platform. Thus we modify the case study for use on a dual-core system. Specifically, we scale the WCET of each task to reach a total utilization of 1.9 (Averagely 95\% for each processor). We apply both the MILP as well as the MIXO-guided optimization to optimize the case study system. We consider various task partition schemes on the two cores to obtain the results as shown in Table 7.3. The first column shows the number of tasks allocated to core 0 and the second column shows the number of tasks allocated to core 1.

While both are capable of terminating and finding the same optimal solution, the MIXOguided optimization technique is 2 to 3 magnitudes faster than MILP. Furthermore, MIXObased approach remains consistent with the varying partitioning scheme, whereas the MILP's complexity drastically increases with more number of tasks on a single core. It is also interesting to note that although the size of the fuel injection case study is larger than the randomly generated systems in the previous experiment, the performance of MIXOguided algorithm is much better. This is mainly due to the following characteristics of the case study: (i) The total utilization of the case study is very high, almost approaching the 


\begin{tabular}{|c|c|c|c|c|c|}
\hline \multicolumn{2}{|c|}{ Task Count } & \multicolumn{2}{|c|}{ Run Time } & \multirow{2}{*}{ Objective } & \multirow{2}{*}{ Utilization } \\
\hline Core 0 & Core 1 & MILP & MIXO-guided & & 0.94 \\
\hline 90 & 0 & $25 \mathrm{hrs} 2 \mathrm{~min}$ & $5.15 \mathrm{sec}$ & 21 & 1.90 \\
\hline 80 & 10 & $3 \mathrm{hrs} 10 \mathrm{~min}$ & $8.45 \mathrm{sec}$ & 21 & 1.90 \\
\hline 70 & 20 & $1 \mathrm{hr} 18 \mathrm{~min}$ & $6.11 \mathrm{sec}$ & 21 & 1.90 \\
\hline 60 & 30 & $26 \mathrm{~min} 45 \mathrm{sec}$ & $6.38 \mathrm{sec}$ & 21 & 1.90 \\
\hline 45 & 45 & $19 \mathrm{~min} 4 \mathrm{sec}$ & $7.7 \mathrm{sec}$ & 21 & \\
\hline
\end{tabular}

Table 7.3: Results for Fuel-Injection Case Study

limit that allows schedulability; (ii) For many of the low rate to high rate communication links $l_{i, j}$, the periods of the reader and writer are drastically different (e.g., a $1 \mathrm{~Hz}$ block communicating to a $250 \mathrm{~Hz}$ block). Since the WCETs of blocks are roughly proportional to their periods, enforcing a partial execution order for these links where the lower rate task executes first would easily cause system unschedulability. As a result, for many of the low rate to high rate communication links, there is only one possible partial execution order that may be enforced. For other high rate to low rate communications $l_{i, j}$, enforcing $f_{i, j}$ is typically the optimal decision as it mostly helps schedulability without having to introduce unit delay blocks. The proposed MIXO-guided optimization technique readily exploits such characteristics. Specifically, since it starts with an overapproximation of the feasibility region (the constraints on $\mathrm{F}$-feasibility is initially omitted), it naturally attempts to enforce $f_{i, j}$ for all communication links $l_{i, j}$, which is typically optimal for high rate to low rate communication. For low rate to high rate communication $l_{i, j}$, the fact that many of them have only one feasible execution order would lead the algorithm to compute lots of MIXOs that contains only one element (i.e., $U=\left\{f_{i, j}\right\}$ ). The implied constraint of such one-element MIXO essentially fixes the value on the variable $l_{i, j}$. This quickly leads the algorithm to reduce the search space and identify the optimal solution. Such problem-specific optimization structure appears to be more difficult to exploit in standard MILP. 


\section{Chapter 8}

\section{Conclusion and Future Work}

In this thesis, we consider the problem of software synthesis for Simulink models on multicore architectures with partitioned fixedpriority scheduling. We consider a mechanism for semantics preservation on such platforms, that assigns task offsets and leverages the Simulink RT blocks. This avoids accessing the global shared variables at the same time from the writer and reader on different cores, and enforces a proper execution order between them. We propose to optimize the cost associated to the unit delay RT block, and present two approaches. One is a direct MILP formulation, the other is a customized exact procedure. Our evaluation on random system shows that for small systems of size less than 50 tasks, the two algorithms performs comparably well. For larger systems however, our customized exact procedure algorithm is much more scalable. For randomly generated systems, our customized procedure runs 10X faster and gives significantly better optimization quality comparing to MILP. Our evaluation on industrial case study also demonstrates its potential for handling large scale real-world problems efficiently as it performs upto four orders of magnitude faster than MILP. Furthermore, our framework shows consistent performance for varying utilization and partitioning schemes, whereas MILP on the other hand shows strong variations. For future work, we plan to include task-to-core allocation into the design space to further improve the solution quality. Furthermore, we seek to include the mapping of blocks to tasks as well to further optimize the generated software quality. To get a more accurate estimate on the improvement in performance, we seek to implement this on a hardware platform in the future (like Kalray 256). 


\section{Bibliography}

[1] Analytics. URL https://www.ibm.com/analytics/data-science/ prescriptive-analytics/cplex-optimizer.

[2] Zaid Al-bayati, Haibo Zeng, Marco Di Natale, and Zonghua Gu. Multitask implementation of synchronous reactive models with earliest deadline first scheduling. In IEEE International Symposium on Industrial Embedded Systems, 2013.

[3] Sebastian Altmeyer, Robert I. Davis, Leandro Indrusiak, Claire Maiza, Vincent Nelis, and Jan Reineke. A generic and compositional framework for multicore response time analysis. In Proceedings of the 23rd International Conference on Real Time and Networks Systems, RTNS '15, pages 129-138, New York, NY, USA, 2015. ACM. ISBN 9781-4503-3591-1. doi: 10.1145/2834848.2834862. URL http://doi.acm.org/10.1145/ 2834848.2834862 .

[4] N. C. Audsley. On priority asignment in fixed priority scheduling. Inf. Process. Lett., 79(1):39-44, May 2001. ISSN 0020-0190. doi: 10.1016/S0020-0190(00)00165-4. URL http://dx.doi.org/10.1016/S0020-0190(00)00165-4.

[5] Philip Axer, Rolf Ernst, Heiko Falk, Alain Girault, Daniel Grund, Nan Guan, Bengt Jonsson, Peter Marwedel, Jan Reineke, Christine Rochange, Maurice Sebastian, Reinhard Von Hanxleden, Reinhard Wilhelm, and Wang Yi. Building timing predictable embedded systems. ACM Trans. Embed. Comput. Syst., 13(4):82:1-82:37, March 2014. ISSN 1539-9087. doi: 10.1145/2560033. URL http://doi . acm.org/10.1145/2560033.

[6] Gérard Berry and Georges Gonthier. The esterel synchronous programming language: Design, semantics, implementation. Sci. Comput. Program., 19(2):87-152, November 
1992. ISSN 0167-6423. doi: 10.1016/0167-6423(92)90005-V. URL http://dx.doi. org/10.1016/0167-6423(92) 90005-V.

[7] Dai Bui, Edward Lee, Isaac Liu, Hiren Patel, and Jan Reineke. Temporal isolation on multiprocessing architectures. In Proceedings of the 48th Design Automation Conference, DAC '11, pages 274-279, New York, NY, USA, 2011. ACM. ISBN 978-1-45030636-2. doi: 10.1145/2024724.2024787. URL http://doi . acm.org/10.1145/2024724. 2024787.

[8] Thomas Carle, Dumitru Potop-Butucaru, Yves Sorel, and David Lesens. From dataflow specification to multiprocessor partitioned time-triggered real-time implementation. Leibniz Transactions on Embedded Systems, 2(2):01-1-01:30, 2015. ISSN 2199-2002. doi: 10.4230/LITES-v002-i002-a001. URL https://ojs.dagstuhl.de/index.php/lites/ article/view/LITES-v002-i002-a001.

[9] P. Caspi, N. Scaife, C. Sofronis, and S. Tripakis. Semantics-preserving multitask implementation of synchronous programs. ACM Trans. Embed. Comput. Syst., 7(2):1-40, 2008.

[10] Paul Caspi and Albert Benveniste. Time-robust discrete control over networked loosely time-triggered architectures. In IEEE Conf. Decision \& Control, 2008.

[11] Paul Caspi, Adrian Curic, Aude Maignan, Christos Sofronis, Stavros Tripakis, and Peter Niebert. From simulink to scade/lustre to tta: A layered approach for distributed embedded applications. In ACM Conference on Language, Compiler, and Tool for Embedded Systems, 2003.

[12] Paul Caspi, Norman Scaife, Christos Sofronis, and Stavros Tripakis. Semanticspreserving multitask implementation of synchronous programs. ACM Transactions on Embedded Computing Systems (TECS), 7(2):15, 2008. 
[13] J. Chen and A. Burns. Loop-free asynchronous data sharing in multiprocessor real-time systems based on timing properties. In IEEE Conference on Real-Time Computing Systems and Applications, 1999.

[14] Robert I Davis and Alan Burns. Priority assignment for global fixed priority pre-emptive scheduling in multiprocessor real-time systems. In Real-Time Systems Symposium, 2009, RTSS 2009. 30th IEEE, pages 398-409. IEEE, 2009.

[15] Robert I. Davis, Sebastian Altmeyer, Leandro Indrusiak, Claire Maiza, Vincent Nelis, and Jan Reineke. An extensible framework for multicore response time analysis. RealTime Systems, 2017.

[16] Marco Di Natale and Valerio Pappalardo. Buffer optimization in multitask implementations of simulink models. ACM Transactions on Embedded Computing Systems, 7(3): $23,2008$.

[17] Marco Di Natale, Liangpeng Guo, Haibo Zeng, and Alberto Sangiovanni-Vincentelli. Synthesis of multitask implementations of simulink models with minimum delays. IEEE Transactions on Industrial Informatics, 6(4):637-651, 2010.

[18] Marco Di Natale, Liangpeng Guo, Haibo Zeng, and Alberto Sangiovanni-Vincentelli. Synthesis of multitask implementations of simulink models with minimum delays. Industrial Informatics, IEEE Transactions on, 6(4):637-651, 2010.

[19] R. P. Dick, D. L. Rhodes, and W. Wolf. Tgff: task graphs for free. In Hardware/Software Codesign, 1998. (CODES/CASHE '98) Proceedings of the Sixth International Workshop on, pages 97-101, Mar 1998. doi: 10.1109/HSC.1998.666245.

[20] Guy Durrieu, Madeleine Faugère, Sylvain Girbal, Daniel Gracia Pérez, Claire Pagetti, and W. Puffitsch. Predictable Flight Management System Implementation on a Mul- 
ticore Processor. In Embedded Real Time Software (ERTS'14), TOULOUSE, France, February 2014. URL https://hal .archives-ouvertes.fr/hal-01121700.

[21] J. Forget, F. Boniol, D. Lesens, and C. Pagetti. A multi-periodic synchronous data-flow language. In IEEE High Assurance Systems Engineering Symposium, Dec 2008.

[22] J. Forget, F. Boniol, and C. Pagetti. Verifying end-to-end real-time constraints on multiperiodic models. In 2017 22nd IEEE International Conference on Emerging Technologies and Factory Automation (ETFA), pages 1-8, Sept 2017. doi: 10.1109/ETFA.2017. 8247612.

[23] Amaury Graillat, Matthieu Moy, Pascal Raymond, and Benoît Dupont De Dinechin. Parallel Code Generation of Synchronous Programs for a Many-core Architecture. In Design, Automation and Test in Europe, 2018.

[24] Nicholas Halbwachs, Paul Caspi, Pascal Raymond, and Daniel Pilaud. The synchronous data flow programming language lustre. Proceedings of the IEEE, 79(9):1305-1320, 1991.

[25] G. Han, H. Zeng, M. Di Natale, X. Liu, and W. Dou. Experimental evaluation and selection of data consistency mechanisms for hard real-time applications on multicore platforms. IEEE Transactions on Industrial Informatics, 10(2):903-918, May 2014. ISSN 1551-3203. doi: 10.1109/TII.2013.2290585.

[26] Donghyun Kang, Junchul Choi, and Soonhoi Ha. Worst case delay analysis of shared resource access in partitioned multi-core systems. In Proceedings of the 15th IEEE/ACM Symposium on Embedded Systems for Real-Time Multimedia, ESTIMedia '17, pages 8492, New York, NY, USA, 2017. ACM. ISBN 978-1-4503-5117-1. doi: 10.1145/3139315. 3139322. URL http://doi.acm.org/10.1145/3139315.3139322.

[27] Timon Kelter and Peter Marwedel. Parallelism analysis: Precise wcet values for complex 
multi-core systems. In Cyrille Artho and Peter Csaba Ölveczky, editors, Formal Techniques for Safety-Critical Systems, pages 142-158, Cham, 2015. Springer International Publishing. ISBN 978-3-319-17581-2.

[28] Enagnon Cédric Klikpo and Alix Munier-Kordon. Preemptive scheduling of dependent periodic tasks modeled by synchronous dataflow graphs. In Proceedings of the 24th International Conference on Real-Time Networks and Systems, RTNS '16, pages 77-86, New York, NY, USA, 2016. ACM. ISBN 978-1-4503-4787-7. doi: 10.1145/2997465. 2997474. URL http://doi.acm.org/10.1145/2997465.2997474.

[29] Simon Kramer, Dirk Ziegenbein, and Arne Hamann. Real world automotive benchmarks for free. In 6th International Workshop on Analysis Tools and Methodologies for Embedded and Real-time Systems (WATERS), 2015.

[30] Cláudio Maia, Marko Bertogna, Luís Nogueira, and Luis Miguel Pinho. Response-time analysis of synchronous parallel tasks in multiprocessor systems. In Proceedings of the 22Nd International Conference on Real-Time Networks and Systems, RTNS '14, pages 3:3-3:12, New York, NY, USA, 2014. ACM. ISBN 978-1-4503-2727-5. doi: 10.1145/ 2659787.2659815. URL http://doi.acm.org/10.1145/2659787.2659815.

[31] Leonardo Mangeruca, Massimo Baleani, Alberto Ferrari, and Alberto SangiovanniVincentelli. Uniprocessor scheduling under precedence constraints for embedded systems design. ACM Transactions on Embedded Computing Systems, 7(1):6, 2007.

[32] Sébastien Martinez, Damien Hardy, and Isabelle Puaut. Quantifying wcet reduction of parallel applications by introducing slack time to limit resource contention. In Proceedings of the 25th International Conference on Real-Time Networks and Systems, RTNS '17, pages 188-197, New York, NY, USA, 2017. ACM. ISBN 978-1-4503-5286-4. doi: 10.1145/3139258.3139263. URL http://doi.acm.org/10.1145/3139258.3139263. 
[33] Vincent Nélis, Patrick Meumeu Yomsi, and Luís Miguel Pinho. The Variability of Application Execution Times on a Multi-Core Platform. In Martin Schoeberl, editor, 16th International Workshop on Worst-Case Execution Time Analysis (WCET 2016), volume 55 of OpenAccess Series in Informatics (OASIcs), pages 6:1-6:11, Dagstuhl, Germany, 2016. Schloss Dagstuhl-Leibniz-Zentrum fuer Informatik. ISBN 978-3-95977025-5. doi: 10.4230/OASIcs.WCET.2016.6. URL http://drops.dagstuhl.de/opus/ volltexte/2016/6899.

[34] Haluk Ozaktas, Christine Rochange, and Pascal Sainrat. Minimizing the cost of synchronisations in the wcet of real-time parallel programs. In Proceedings of the 17th International Workshop on Software and Compilers for Embedded Systems, SCOPES '14, pages 98-107, New York, NY, USA, 2014. ACM. ISBN 978-1-4503-2941-5. doi: 10.1145/2609248.2609261. URL http://doi.acm.org/10.1145/2609248.2609261.

[35] Claire Pagetti, Julien Forget, Frédéric Boniol, Mikel Cordovilla, and David Lesens. Multi-task implementation of multi-periodic synchronous programs. Discrete event $d y$ namic systems, 21(3):307-338, 2011.

[36] Claire Pagetti, David Saussié, Romain Gratia, Eric Noulard, and Pierre Siron. The ROSACE case study: From simulink specification to multi/many-core execution. In IEEE Real-Time and Embedded Technology and Applications Symposium, 2014.

[37] Q. Perret, P. Maurere, E. Noulard, C. Pagetti, P. Sainrat, and B. Triquet. Temporal isolation of hard real-time applications on many-core processors. In 2016 IEEE RealTime and Embedded Technology and Applications Symposium (RTAS), pages 1-11, April 2016. doi: 10.1109/RTAS.2016.7461363.

[38] D. Potop-Butucaru, B. Caillaud, and A. Benveniste. Concurrency in synchronous systems. In Int. Conf. Application of Concurrency to System Design, 2004. 
[39] D. Potop-Butucaru, S. A. Edwards, and G. Berry. Compiling Esterel. Springer, 2007.

[40] Dumitru Potop-Butucaru and Isabelle Puaut. Integrated Worst-Case Execution Time Estimation of Multicore Applications. In Claire Maiza, editor, 13th International Workshop on Worst-Case Execution Time Analysis, volume 30 of OpenAccess Series in Informatics (OASIcs), pages 21-31, Dagstuhl, Germany, 2013. Schloss Dagstuhl-LeibnizZentrum fuer Informatik. ISBN 978-3-939897-54-5. doi: 10.4230/OASIcs.WCET.2013. 21. URL http://drops .dagstuhl.de/opus/volltexte/2013/4119.

[41] Claudius Ptolemaeus, editor. System Design, Modeling, and Simulation using Ptolemy II. Ptolemy.org, 2014. URL http://ptolemy.org/books/Systems.

[42] W. Puffitsch, E. Noulard, and C. Pagetti. Mapping a multi-rate synchronous language to a many-core processor. In IEEE Real-Time and Embedded Technology and Applications Symposium, April 2013.

[43] Wolfgang Puffitsch, Eric Noulard, and Claire Pagetti. Off-line mapping of multi-rate dependent task sets to many-core platforms. Real-Time Systems, 51(5):526-565, 2015.

[44] Hamza Rihani, Matthieu Moy, Claire Maiza, Robert I. Davis, and Sebastian Altmeyer. Response Time Analysis of Synchronous Data Flow Programs on a Many-Core Processor. In RTNS 2016, 24th International Conference on Real-Time Networks and Systems, Brest, France, Oct 2016.

[45] The MathWorks. The mathworks simulink and stateflow user's manuals. web page: http://www.mathworks.com.

[46] S. Tripakis, C. Pinello, A. Benveniste, A. Sangiovanni-Vincent, P. Caspi, and M. Di Natale. Implementing synchronous models on loosely time triggered architectures. IEEE Transactions on Computers, 57(10):1300-1314, Oct 2008. 
[47] Stavros Tripakis, Christos Sofronis, Paul Caspi, and Adrian Curic. Translating discretetime simulink to lustre. ACM Transactions on Embedded Computing Systems (TECS), 4(4):779-818, 2005.

[48] Simon Wegener. Towards Multicore WCET Analysis. In Jan Reineke, editor, 17th International Workshop on Worst-Case Execution Time Analysis (WCET 2017), volume 57 of OpenAccess Series in Informatics (OASIcs), pages 7:1-7:12, Dagstuhl, Germany, 2017. Schloss Dagstuhl-Leibniz-Zentrum fuer Informatik. ISBN 978-3-95977-057-6. doi: 10.4230/OASIcs.WCET.2017.7. URL http://drops.dagstuhl.de/opus/volltexte/ $2017 / 7311$.

[49] Haibo Zeng and Marco Di Natale. Mechanisms for guaranteeing data consistency and flow preservation in autosar software on multi-core platforms. In IEEE International Symposium on Industrial Embedded Systems, 2011.

[50] Haibo Zeng and Marco Di Natale. Mechanisms for guaranteeing data consistency and flow preservation in autosar software on multi-core platforms. In 6th IEEE International Symposium on Industrial Embedded Systems, pages 140-149, 2011.

[51] Y. Zhao and H. Zeng. The virtual deadline based optimization algorithm for priority assignment in fixed-priority scheduling. In 2017 IEEE Real-Time Systems Symposium (RTSS), volume 00, pages 116-127, Dec 2017. doi: 10.1109/RTSS.2017.00018. URL doi . ieeecomputersociety.org/10.1109/RTSS . 2017.00018.

[52] Yecheng Zhao and Haibo Zeng. The concept of unschedulability core for optimizing priority assignment in real-time systems. In Proceedings of the Conference on Design, Automation $8 \mathcal{G}$ Test in Europe, DATE '17, pages 232-237, 3001 Leuven, Belgium, Belgium, 2017. European Design and Automation Association. URL http: //dl.acm.org/citation. cfm?id=3130379.3130432. 